\title{
Comparison of six simulation codes for positive streamers in air
}

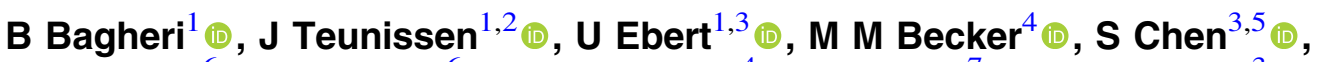

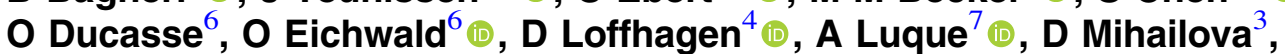 \\ J M Plewa ${ }^{6}$, J van Dijk ${ }^{3}$ and M Yousfi $^{6}$ (i) \\ ${ }^{1}$ Centrum Wiskunde \& Informatica, Amsterdam, The Netherlands \\ ${ }^{2}$ Centre for Mathematical Plasma-Astrophysics, KU Leuven, Belgium \\ ${ }^{3}$ Technical University of Eindhoven, Eindhoven, The Netherlands \\ ${ }^{4}$ Leibniz Institute for Plasma Science and Technology (INP), Greifswald, Germany \\ ${ }^{5}$ College of Electrical and Information Engineering, Hunan University, Changsha, People's Republic of \\ China \\ ${ }^{6}$ LAPLACE, Université de Toulouse, CNRS, INPT, UPS, France \\ ${ }^{7}$ Instituto de Astrofisica de Andalucia (IAA-CSIC), Granada, Spain \\ E-mail: bagheri@cwi.nl
}

Received 4 April 2018, revised 10 July 2018

Accepted for publication 1 August 2018

Published 13 September 2018

\begin{abstract}
We present and compare six simulation codes for positive streamer discharges from six different research groups. Four groups use a fully self-implemented code and two make use of COMSOL Multiphysics ${ }^{\circledR}$. Three test cases are considered, in which axisymmetric positive streamers are simulated in dry air at 1 bar and $300 \mathrm{~K}$ in an undervolted gap. All groups use the same fluid model with the same transport coefficients. The first test case includes a relatively high background density of electrons and ions without photoionization. When each group uses their standard grid resolution, results show considerable variation, particularly in the prediction of streamer velocities and maximal electric fields. However, for sufficiently fine grids good agreement is reached between several codes. The second test includes a lower background ionization density, and oscillations in the streamer properties, branching and numerical instabilities are observed. By using a finer grid spacing some groups were able to reach reasonable agreement in their results, without oscillations. The third test case includes photoionization, using both Luque's and Bourdon's Helmholtz approximation. The results agree reasonably well, and the numerical differences appear to be more significant than the type of Helmholtz approximation. Computing times, used hardware and numerical parameters are described for each code and test case. We provide detailed output in the supplementary data, so that other streamer codes can be compared to the results presented here.
\end{abstract}

Keywords: positive streamer discharge, simulation comparison, photoionization

\section{Introduction}

Streamer discharges [1-3] are rapidly growing ionized channels. Their growth is caused by strong electric field enhancement at their tips, which allows them to propagate in regions where the background electric field is below the breakdown threshold. Streamers are the precursors of sparks, arcs and lightning leaders, and they are also used in diverse applications in plasma technology, such as surface processing [4], ignition and combustion [5], flow control [6], environmental applications [7, 8], catalysis [9], sterilization and disinfection [10], or the production of free radicals [11]. For medical applications such as wound healing [12], streamers are commonly applied in the form of atmospheric pressure plasma jets [13, 14].

Streamer simulations can be computationally and numerically challenging due to their steep density gradients and strongly nonlinear coupling with the electric field. Early work on streamer modeling includes, for example, [1, 15-18]. Like these ones, most models developed since have been of the plasma fluid type, in which electron and ion densities 
evolve in time. Transport and reaction coefficients are used as input data; they can be computed with a Boltzmann solver such as Bolsig + [19]. The fluid equations are often solved on structured grids using a finite volume discretization. Different numerical methods have been used to ensure that the solutions are stable. The use of flux/slope limiters is quite common in recent work [20-22, 22-26], and a high-order WENO scheme was used in [27]. Other methods such as the Scharfetter-Gummel scheme [28-30] and the flux-corrected transport method [31, 32] have also been used.

An important development has been the use of adaptive mesh refinement, so that a fine mesh is only placed where it is required, see for example [20, 24, 25, 33, 34]. Structured-grid models can be adapted to more complex geometries [35], but this is often challenging. This has motivated the development of finite element discharge simulations on unstructured grids, see e.g. [36-40]. An alternative way to handle complex geometries is to apply finite volume methods to unstructured grids, as is done with the nonPDPSIM code [41, 42].

Besides fluid models, particle-in-cell (PIC) codes can also be used to simulate streamer discharges, see e.g. [43-47]. In PIC codes, the velocity distribution function $f(\mathbf{x}, \mathbf{v}, t)$ of any plasma species is determined by solving the equations of motion for the particles, which makes them computationally quite expensive. Hybrid models can alleviate this by coupling PIC and fluid models [48, 49].

\subsection{Verification and validation}

Computer simulations can be a valuable tool in the study of physical systems, but their results are not always fully correct. In recent years, the verification and validation $(\mathrm{V} \& \mathrm{~V})$ of simulation codes [50] has received increasing attention in the discharge physics community, instigated by the work of [51]. Code verification checks whether the 'conceptual model' (e.g. the underlying equations and their discretization) is correctly implemented. Code validation checks whether the code's predictions are in sufficiently good agreement with real experiments.

In this paper, we work towards code verification. For individual model components, such as the field solver, analytic solutions can be used for verification, but for a full multidimensional streamer model no analytic solutions exist. We therefore compare different codes that implement the same underlying equations, with the aim of finding numerical convergence for sufficiently fine grids and time steps. When this occurs, the results can be used as a benchmark for other codes, which is one of the goals of this study. Other goals are to spot bugs or a lack of convergence in the codes, to show the effects of numerical discretization on physical predictions, and to shed insight on the (dis)advantages of different numerical discretizations.

There have been several comparisons of streamer models. Particle, fluid and hybrid models were extensively compared for different discharge applications in [52]. In [53], particle, fluid and hybrid models were compared for a negative streamer in $3 \mathrm{D}$, and in [54] a particle model was compared to three fluid models for 'one-dimensional streamers' (ionization waves). In [37], a finite element model was compared to a finite volume model, for a positive streamer in an axisymmetric point-to-plane geometry. Three different approximations for photoionization (see section 2.3) were systematically compared in [55], which also includes the axisymmetric simulations of positive streamers. Furthermore, there have also been quite a few studies in which experimental results are compared with simulations; examples include [56-58].

\subsection{Choice of discharge model}

Most streamer simulations are performed with fluid models, probably because particle-in-cell simulations are often computationally too expensive. In the present study, we therefore compare the implementations of plasma fluid models from six different groups. Even when only considering fluid models, such models differ in their constituting equations, dimensionality (2D, axisymmetric, 3D), type of grid and grid refinement, and their spatial/temporal discretization. To restrict the scope of this study, all groups use an axisymmetric plasma fluid model of the drift-diffusion-reaction type here, in combination with the local field approximation. This type of model is commonly used because of its simplicity and robustness. A further reason to opt for axisymmetric models is that they are computationally the cheapest ones that can be used to simulate the spatiotemporal development of individual streamers. Full 3D simulations are computationally much more expensive, and 1D models cannot self-consistently describe the electric field enhancement of a streamer channel.

\subsection{Test cases}

We consider three test cases for positive streamers in dry air at $1 \mathrm{bar}$ and $300 \mathrm{~K}$, in which positive streamers form more easily than negative ones [59]. To separate numerical differences from those in the input data, we use analytic expressions for the transport coefficients, as described in section 2.2.

The computational domain, boundary conditions and initial conditions are kept simple, so that different codes can easily implement them. Discharges are simulated between planar electrodes in a square domain with a radius and height of $1.25 \mathrm{~cm}$. A background electric field of $15 \mathrm{kV} \mathrm{cm}^{-1}$ is present, which is well below the breakdown value. To locally enhance the electric field to values above the breakdown threshold, a Gaussian distribution of positive ions is placed below the anode. In contrast to e.g., a needle electrode, for example, such a Gaussian seed can easily be implemented in a discharge model, and the smooth Gaussian profile helps to reduce differences in the discretization of the initial condition. A complete description of the simulation conditions is given in section 2.4.

A drawback of the simplified conditions used in this study is that they are quite far from typical experimental conditions. We use simplified conditions here, because we were aiming towards code verification, which is by itself 
already quite challenging for streamer models. Future validation efforts could include more realistic electrode geometries, voltage rise times, repeated pulses, plasma chemistry, etc, and, of course, fully 3D simulations to capture events with multiple streamers.

A positive streamer needs free electrons ahead of it in order to propagate. Three test cases are considered in which the source of such free electrons is varied, and thereby also the streamer properties. The first two test cases use a constant initial background density of electrons and ions instead of photoionization. In the first case, this density has quite a high value of $10^{13} \mathrm{~m}^{-3}$. In the second case this density is $10^{9} \mathrm{~m}^{-3}$, which creates much steeper gradients in the ionization front, making it computationally quite challenging. The idea behind these two cases is that most streamer codes should be able to handle the first case, and that the second case can shed more light on the numerical properties and robustness of the code.

The third test case is identical to the second one, except that photoionization is included, based on Zhelenznyak's model [60], see section 2.3. This case therefore reflects a more typical simulation in air, in which photoionization is always present.

It was not possible for each participating group to perform a convergence study for each test case, due to time and computational constraints. Instead, groups first performed simulations with their 'standard' numerical parameters (e.g. mesh spacing, time step). Afterwards, groups that were able to do so performed additional simulations aiming at numerical convergence, using finer grids and smaller time steps.

\subsection{Other possible tests of streamer simulations}

Here we focus on an already quite complex problem, namely on an axisymmetric positive streamer in air. Alternatively, one could have started with a one-dimensional planar ionization front as in [54], for example. However, in 1D there is no electric field enhancement, so the electric field ahead of the front is constant. This field needs to be above the breakdown value to support a discharge, but then photoionization or background ionization lead to continuous ionization growth, ultimately causing a loss of the front structure. Only negative ionization fronts without photoionization or background ionization (e.g. in pure nitrogen) create a planar front that is physically realistic and that approaches a constant speed. Simulation results on such fronts can be compared to analytic expressions [61, 62], which together with the reduced computational cost of 1D models make it easier to establish numerical convergence.

Furthermore, one can think of tests using the method of manufactured solutions [63], although we have not yet been able to construct a good test case. For $2 \mathrm{D}$ and $3 \mathrm{D}$ problems, computing source terms for the manufactured solution can become quite complicated due to the coupling with Poisson's equation, which is less of an issue in 1D.

\subsection{Organization of the paper}

The paper is organized as follows. In section 2, the definition of the discharge model is presented, along with the analytical transport coefficients. In section 3 , the numerical implementation of this model by each group is described. The three test cases are presented in sections 4-6. Each section contains the results of the different groups and a discussion of the observed differences. We conclude with a general discussion of the results of the comparison in section 7. A detailed description of photoionization is provided in appendix A. Note that the paper also contains supplementary material that can, for example, be used to test new models; this material is described in appendix B.

\section{Discharge model and simulation conditions}

\subsection{Model equations}

Here we compare different implementations of a drift-diffusion-reaction type fluid model, where the transport of ions in the short time-scale of streamer propagation is neglected. In such a model, the electron density $n_{e}$ and positive ion density $n_{i}$ evolve in time as

$$
\begin{gathered}
\partial_{t} n_{e}=\nabla \cdot\left(n_{e} \mu_{e} \mathbf{E}+D_{e} \nabla n_{e}\right)+\bar{\alpha} \mu_{e}|\mathbf{E}| n_{e}+S_{\mathrm{ph}}, \\
\partial_{t} n_{i}=\bar{\alpha} \mu_{e}|\mathbf{E}| n_{e}+S_{\mathrm{ph}},
\end{gathered}
$$

where $\mu_{e}$ is the (positive) electron mobility, $D_{e}$ is the electron diffusion coefficient, $\bar{\alpha}$ is the effective ionization coefficient (ionization $\alpha$ minus attachment $\eta$ ), $\mathbf{E}$ is the electric field, and $S_{\mathrm{ph}}$ is the nonlocal photoionization term. For the transport coefficients, the local field approximation is used, so that $\bar{\alpha}$, $\mu_{e}$ and $D_{e}$ depend on the local electric field strength. Ions are assumed to be immobile. The electric field is computed in electrostatic approximation according to

$$
\begin{gathered}
\mathbf{E}=-\nabla \phi, \\
\nabla^{2} \phi=-\frac{e\left(n_{i}-n_{e}\right)}{\epsilon_{0}},
\end{gathered}
$$

where $\phi$ is the electric potential, $\epsilon_{0}$ is the vacuum permittivity and $e$ is the elementary charge.

\subsection{Transport coefficients}

We consider streamer discharges in dry air, containing $80 \% \mathrm{~N}_{2}$ and $20 \% \mathrm{O}_{2}$, at $p=1$ bar and $T=300$ Kelvin. To separate the numerical differences from those in the input data, all compared codes use the analytical approximations for the coefficients from [64, 65]. The electron drift velocity and electron diffusion coefficient can then be expressed in terms of the reduced electric field $\tilde{E}=(|\mathbf{E}| / N) /(1 \mathrm{Td})$ as

$$
\begin{gathered}
v_{e}=4.2 \times 10^{3} \mathrm{~m} \mathrm{~s}^{-1} \times \tilde{E}^{0.74}, \\
D_{e}=9.7 \times 10^{23} \mathrm{~m}^{-1} \mathrm{~s}^{-1} \times\left(\tilde{E}^{0.22} / N\right),
\end{gathered}
$$

where $N$ is the gas number density and the Townsend unit is $1 \mathrm{Td}=10^{-21} \mathrm{~V} \mathrm{~m}^{2}$. Here we assume $N=p /\left(k_{\mathrm{B}} T\right) \approx$ 
$2.414 \times 10^{25} \mathrm{~m}^{-3}$ where the Boltzmann constant is $k_{\mathrm{B}}$. The electron mobility follows from equation (5) and is given by $\mu_{e}=v_{e} /|\mathbf{E}|$. The Townsend ionization coefficient $(\bar{\alpha})$ is taken from [65]. In our notation, for dry air at 1 bar it is given by

$$
\bar{\alpha}=750 \frac{T_{0}}{T}\left[c_{1}\left(1+\frac{c_{2}}{\left(\frac{|\mathbf{E}|}{750} \frac{T}{T_{0}}\right)^{3}}\right) \exp \frac{c_{3}}{\frac{|\mathbf{E}|}{750} \frac{T}{T_{0}}}-\psi\right],
$$

with $c_{1}=1.75 \times 10^{3}, \quad c_{2}=1.15 \times 10^{12}, \quad c_{3}=-4 \times 10^{4}$ and

$$
\psi=\frac{0.9}{\left[1.49+\exp \left(-\frac{750}{587} \frac{T_{0}}{T}\right)\right]},
$$

and $\bar{\alpha}$ is given in units of $1 \mathrm{~m}^{-1}$. In these equations $T$ is the gas temperature in Kelvin, $T_{0}=273 \mathrm{~K}$, and $|\mathbf{E}|$ is the electric field strength in $\mathrm{V} \mathrm{m}^{-1}$. For the temperature and pressure used in this study, the transport coefficients can be numerically written (omitting all SI units) as

$$
\begin{aligned}
\mu_{e} & =2.3987 E^{-0.26} \\
v_{e} & =2.3987 E^{0.74} \\
D_{e} & =4.3628 \times 10^{-3} E^{0.22} \\
\bar{\alpha} & =\alpha-\eta \\
\alpha & =\left(1.1944 \times 10^{6}+4.3666 \times 10^{26} / E^{3}\right) \mathrm{e}^{-2.73 \times 10^{7} / E} \\
\eta & =340.75,
\end{aligned}
$$

where $E$ is the electric field strength in $\mathrm{V} \mathrm{m}^{-1}, \mu_{e}$ is the mobility in $\mathrm{m}^{2} \mathrm{~V}^{-1} \mathrm{~s}^{-1}$, etc, figure 1 shows the electron drift velocity (top), diffusion coefficient (middle) and net ionization coefficient (bottom) as a function of the electric field. Note that the critical field is about $24 \mathrm{kV} \mathrm{cm}^{-1}$ using these coefficients, whereas a typical value for air is about $30 \mathrm{kV} \mathrm{cm}^{-1}$.

\subsection{Photoionization}

For this comparison study, the photoionization source term is computed according to Zheleznyak's model [60], but using a set of Helmholtz differential equations [55, 66]. A detailed description of the equations and the parameters is given in appendix A.

\subsection{Simulation domain and initial conditions}

An axisymmetric domain is used with $r_{\max }=z_{\max }=$ $1.25 \mathrm{~cm}$, as shown in figure 2. A potential of $\phi_{0}=18.75 \mathrm{kV}$ is applied at $z=1.25 \mathrm{~cm}$, and the boundary at $z=0 \mathrm{~cm}$ is grounded $(\phi=0)$. This gives rise to a homogeneous background electric field of $-15 \mathrm{kV} \mathrm{cm}^{-1}$, which is well below the breakdown field. Homogeneous Neumann boundary conditions $\left(\partial_{r} \phi=0\right)$ are applied to the electric potential at $r=r_{\text {max }}$, and $r=0$. Homogeneous Neumann boundary conditions are also applied to the electron density at all
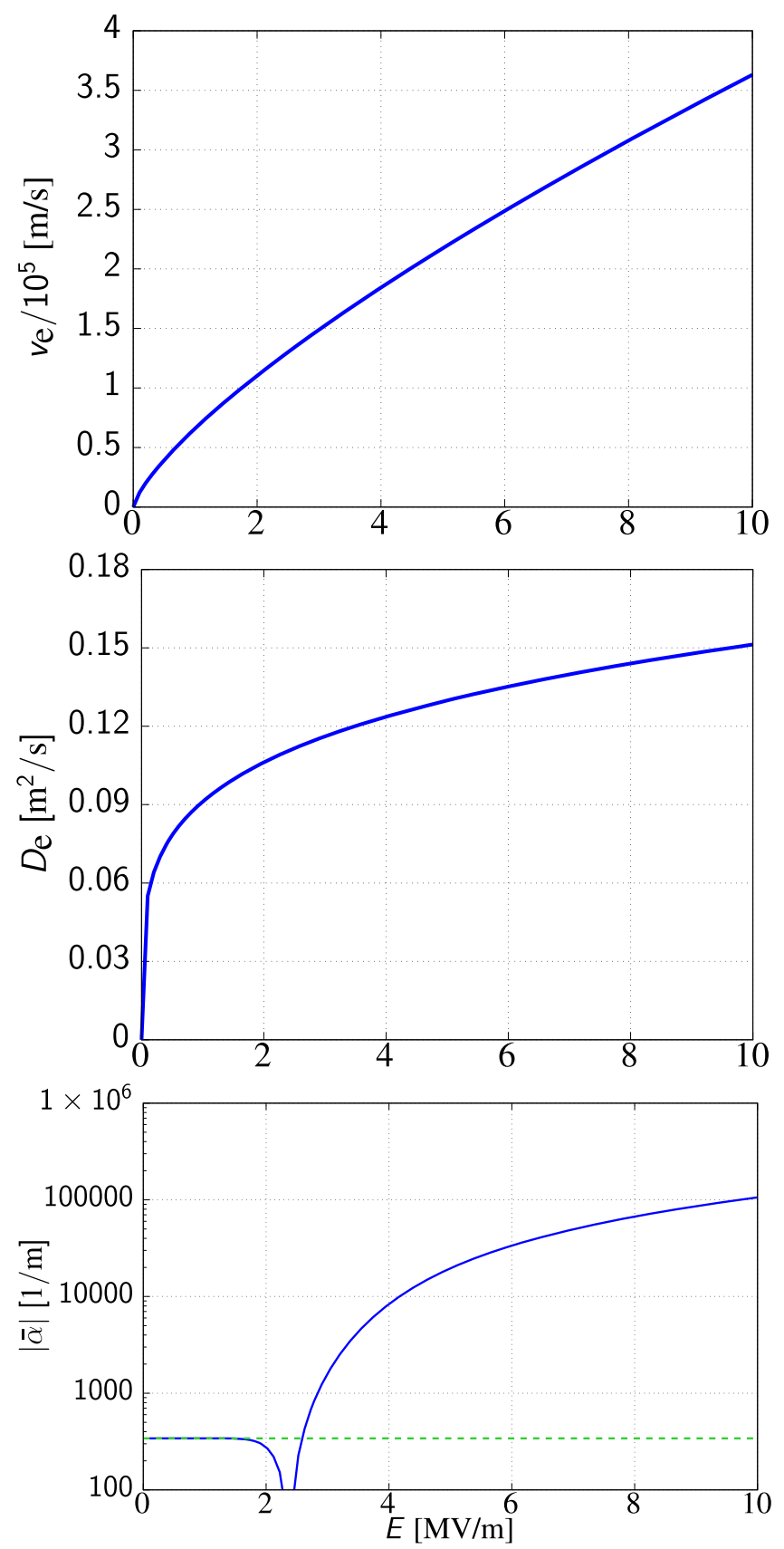

Figure 1. The electron drift velocity (top), diffusion coefficient (middle), and absolute value of the net ionization coefficient $\bar{\alpha}=\alpha-\eta$ (bottom) as a function of the electric field in dry air at 1 bar and 300 Kelvin according to $[64,65]$. The dashed green line in the bottom panel shows the constant attachment rate $\eta$.

boundaries. To locally enhance the electric field, a Gaussian seed of positive ions is included:

$$
n_{i}(r, z)=N_{0} \exp \left[-\frac{r^{2}+\left(z-z_{0}\right)^{2}}{\sigma^{2}}\right],
$$

where $N_{0}=5 \times 10^{18} \mathrm{~m}^{-3}, \quad \sigma=0.4 \mathrm{~mm}$ and $z_{0}=1 \mathrm{~cm}$. Figure 2 shows the resulting initial field configuration. 


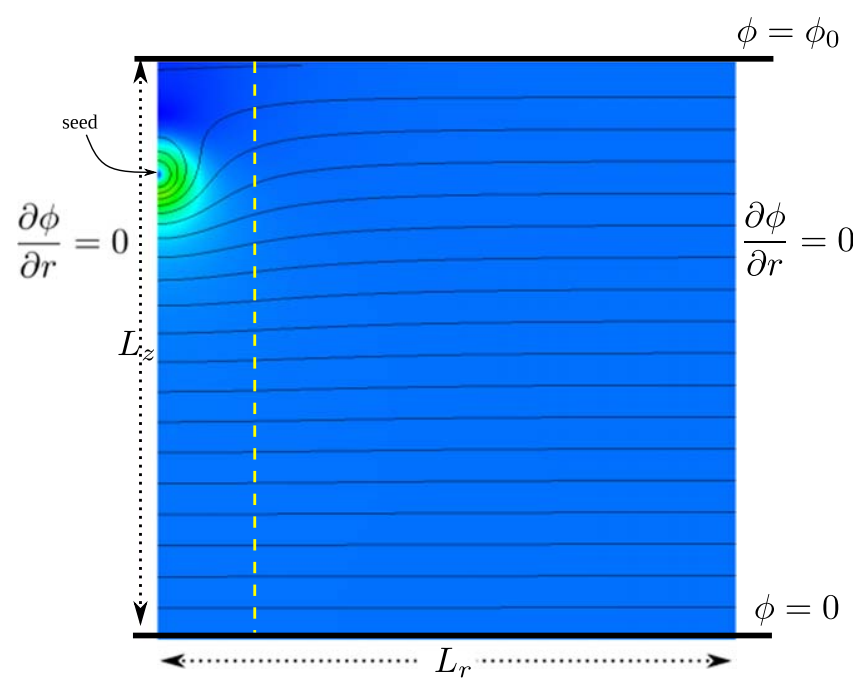

Figure 2. The initial electric field configuration with equipotential lines, where $\phi_{0}=18.75 \mathrm{kV}$ and $L_{z}=L_{r}=1.25 \mathrm{~cm}$. For the electric potential Neumann zero boundary $\left(\partial_{r} \phi=0\right)$ conditions are applied at $r=0$ (due to symmetry) and at $r=1.25 \mathrm{~cm}$. Neumann zero boundary conditions are implemented for the electron density on all boundaries. Instead of a needle electrode, a positive immobile seed with a Gaussian distribution at $(r, z)=(0,1) \mathrm{cm}$ is included. The dashed white line indicates a radial domain up to $2 \mathrm{~mm}$, which is shown in figures 3,9 and 17 .

Homogeneous background ionization levels of $n_{i}=$ $n_{e}=10^{13} \mathrm{~m}^{-3}$ in the first test case and $n_{i}=n_{e}=10^{9} \mathrm{~m}^{-3}$ in the second and third test cases are used.

\section{Description of the codes}

Six groups participate in this study and each group uses a different simulation code. The codes are of the finite volume or finite element type, and in the latter case they are based on the commercial finite element multiphysics framework (COMSOL Multiphysics ${ }^{\circledR}$, www.comsol.com). Some groups use adaptive mesh refinement (AMR) while others use a fixed numerical grid. By using AMR the thin space charge layer around the streamer head and regions with high electric field can be resolved while keeping the computational cost relatively low. In the following, a brief description of the programs and their numerical implementation is provided. The abbreviations for the groups are used in the figures, tables and their captions. A summary of the codes is provided in table 1.

\subsection{Group CWI: B Bagheri, J Teunissen, U Ebert, The Netherlands}

Afivo-streamer [25] www.gitlab.com/MD-CWI-NL/afivostreamer was used, which is an open source code for the plasma fluid simulation of streamer discharges. Afivostreamer is based on the Afivo framework [67], and it employs AMR, geometric multigrid methods for Poisson's equation and OpenMP parallelism. The finite volume method implemented in this code is second order accurate in space and time [25], using a flux limiter and the explicit trapezoidal rule. A fixed numerical grid can be used by changing the refinement routine.

\subsection{Group ES: A Luque, Spain}

ARCoS [68] www.cwimd.nl/doku.php?id=codes:arcos, which is an open source code for the simulation of streamer discharges based on the plasma fluid model, is employed. The code uses AMR and the Poisson equation is solved based on the FISHPACK library. For the simulations in this paper, a constant time step of $1 \mathrm{ps}$ is used. Time-marching is implemented with a second order Runge-Kutta, also called the 'midpoint' rule.

\subsection{Group FR: O Eichwald, O Ducasse, J M Plewa, M Yousfi, France}

The code as implemented in [22] is used. It is a finite volume code (assuming a revolution symmetry around the axis of the streamer propagation) with second order accuracy in time and space. The elliptic equations (for potential and photoionization calculations) are solved with a direct method (noniterative) based on an FFT technique along the axial direction and a cyclic reduction technique along the radial direction. It is worth noting that the use of FFT techniques require a uniform discretization of the axial direction with the total number of mesh cells equal to $2^{n}$ (in our case $n$ varies from 11 to 14 , which leads to a number of mesh cells ranging from 2048 to 16384 in the axial direction). The calculation of the diffusion and drift fluxes of charged species is corrected with the second order MUSCL superbee flux limiter, which can be used without the time splitting technique. Time steps are adapted using the Courant-Friedrichs-Lewy (CFL) condition, which verifies that $\Delta t=C \min \left(\left\{\Delta r / v_{r}\right\},\left\{\Delta z / v_{z}\right\}\right)$ where $\{\ldots\}$ represents the set of possible values. In all the simulations, the CFL number was set to $C=0.1$.

\subsection{Group CN: S Chen, China}

The streamer model is implemented in COMSOL Multiphysics 5.3 and the plasma module is used. In order to avoid negative charge densities, a logarithmic representation of the drift-diffusion equations is employed. A feature called 'source stabilization' is used, which adds a source term $R_{e}=N_{A} \exp \left(-\zeta \ln \left(n_{e}\right)\right)$ to the electron density, where $\zeta=0.4$ is a user-defined tuning parameter. This source term prevents the electron density from approaching zero, and it becomes negligible for high electron densities (reference: the plasma module user's guide, Comsol Multiphysics, pp 207). More time is required to optimize the model's numerical parameters. The backward differentiation formula (BDF) method, with a maximum BDF order 2 and minimum BDF order 1 , is used for time integration. The relative tolerance was set to $10^{-3}$ for all cases. A parallel sparse direct solver (PARDISO) is chosen as the direct linear system solver. 
Table 1. A summary of the codes: FV stands for finite volume, FE for finite element. The DE, FR, TUE and CN groups directly used analytical approximations for the transport coefficients in their code, whereas CWI and ES used tabulated coefficients (provided in the supplements). Please read sections 2.2 and 3 for details.

\begin{tabular}{lcccccc}
\hline & CWI & ES & FR & CN & TUE & DE \\
\hline Finite volume/element & FV & FV & FV & FE (COMSOL) & FV & FE (COMSOL) \\
Unstructured grid & & & & $\checkmark$ & & $\checkmark$ \\
Spatial discretization order & 2 & 2 & 2 & 1 & 2 & 1 \\
Implicit time discretization & & & & $\checkmark$ & $\checkmark$ & $\checkmark$ \\
Time discretization order & 2 & 2 & 2 & 1 to 2 & 1 & 1 to 5 \\
Mesh refinement & $\checkmark$ & $\checkmark$ & $\checkmark$ & $\checkmark$ & $\checkmark$ & $\checkmark$ \\
Adaptive refinement & $\checkmark$ & $\checkmark$ & & $\checkmark$ & & $\checkmark$ \\
Parallel & $\checkmark$ & & $\checkmark$ & $\checkmark$ & & \\
Tabulated transport coefficients & $\checkmark$ & $\checkmark$ & & & & \\
\hline
\end{tabular}

\subsection{Group TUE: D Mihailova, J van Dijk, The Netherlands}

For this study the drift-diffusion module of the plasma simulation software PLASIMO [69] www.plasimo.phys.tue.nl is used. PLASIMO uses a finite volume scheme for the discretization of the quantities of interest. Time integration is done using a Gummel scheme, in which the variables are solved consecutively. The code uses implicit time stepping and an automatically adjusted time step. The semi-implicit treatment of the Poisson equation due to Ventzek et al $[69,70]$ was used to allow time steps beyond the Maxwellian relaxation time of the plasma.

\subsection{Group DE: M M Becker, D Loffhagen, Germany}

At INP, a MATLAB-COMSOL toolbox (MCPlas) has been developed, which uses the MATLAB interface to COMSOL for the automated implementation of the fluid models of gas discharge plasmas in the metal electrode and dielectric barrier discharge configurations described in [71, 72]. This MCPlas toolbox eliminates the restrictions of the COMSOL plasma module and particularly allows the implementation of plasma models in 1D and 2D geometries using extensive reaction kinetics in COMSOL with minimal effort. Recently, MCPlas has been used to investigate plasma generation and thin film deposition by a nonthermal plasma jet at atmospheric pressure [73]. For the present comparison study, the COMSOL model provided by MCPlas has been adapted to meet the predefined benchmark conditions. Linear Lagrange elements (first order spatial discretization) were used to solve the equations. The BDF method provided by COMSOL for time integration was used. Here, the calculation of the time step size is based on an error estimation and the absolute and relative tolerances were both set to $10^{-4}$ for all cases. The maximum BDF order was set to 5 and the minimum BDF order to 1 . Therefore, the time discretization order is automatically adapted between these values depending on the smoothness of the numerical solution.

\section{First test case}

\subsection{Description}

The first test case was designed so that it could be performed by all groups. For simplicity, photoionization is not included. Instead, a homogeneous background ionization level of $n_{i}=n_{e}=10^{13} \mathrm{~m}^{-3}$ is used. Such high background electron densities have frequently been used in streamer simulations, and can be realized in repetitive discharge mode, for example. A high background electron density smooths gradients in the electron and ion density and reduces the electric field at the streamer tip, which makes the simulations easier to perform from a computational point of view. Initial field enhancement is provided by the Gaussian seed of positive ions described in section 2.4, see figure 2. An example of the resulting discharge evolution is shown in figure 3 .

\subsection{Simulation information}

Simulation information, such as grid resolution, used hardware and computation time, is provided below for all participating groups. A summary of simulation settings is provided in tables 2 and 3 .

4.2.1. CWI. Hardware: all the simulations (and test cases) were run on eight threads using an Intel(R) Xeon(R) CPU E31271 v3@3.60 GHz.

Figures 3-7: an AMR grid with a minimal grid size of $\Delta r=\Delta z=3.052 \mu \mathrm{m}$ is used. The maximal number of cells in the simulation domain is 101824 . We use a similar refinement criterion as in [25]: refine if $\alpha(1.0 E) \Delta x>0.8$ and de-refine if $\alpha(1.0 E) \Delta x<0.8$, where $\alpha(E)$ is the fielddependent ionization coefficient, $E$ is the electric field strength in $\mathrm{V} \mathrm{m}^{-1}$ and $\Delta x$ is the cell spacing. The simulations took around $5 \mathrm{~min}$.

Figure 7: the subscripts indicate a constant grid size of $\Delta r=\Delta z=0.763 \mu \mathrm{m} \quad\left(\mathrm{CWI}_{0.7}\right), \quad 1.526 \mu \mathrm{m} \quad\left(\mathrm{CWI}_{1.5}\right) \quad$ and $3.052 \mu \mathrm{m}\left(\mathrm{CWI}_{3.0}\right)$ until $r=500 \mu \mathrm{m}$. The grid is coarsened 

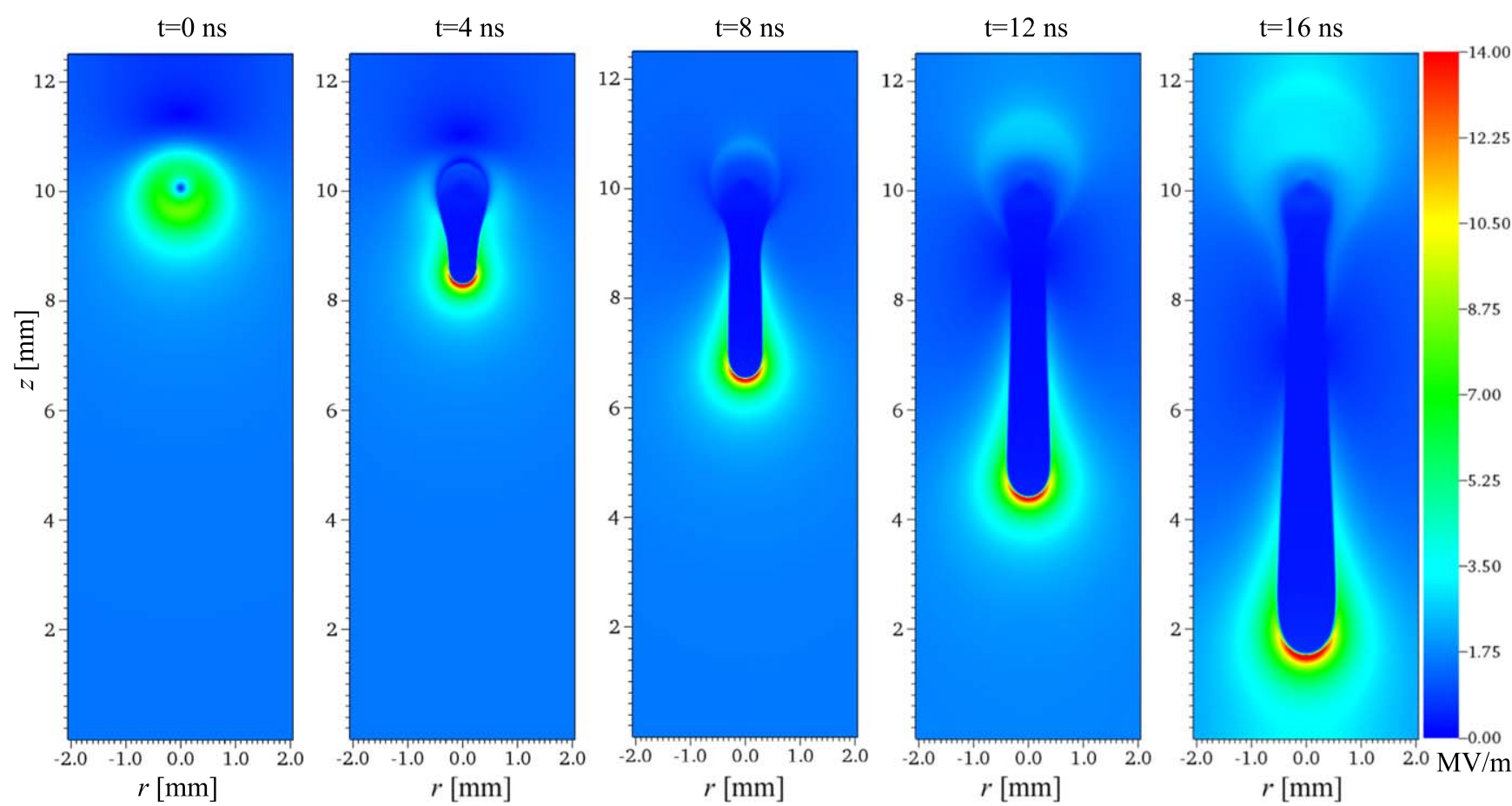

Figure 3. Case 1: a positive streamer propagates downwards. The electric field strength $|\mathbf{E}|$ is shown for different times using data from the CWI group, see section 4.3 and table 2 . The radial direction extends up to $1.25 \mathrm{~cm}$ but is only shown up to $2 \mathrm{~mm}$, as indicated by the dashed line in figure 2.

Table 2. A summary of simulation settings for case 1, figures 5 and 6 . We refer to this setting as 'standard' grid resolution for case 1 in the text. Please read section 4.2 for more details. Note that for DE and $\mathrm{CN}$, the min and max grid size are specified in the narrow rectangular region where the streamer propagates, and not in the whole simulation domain. NA: not available.

\begin{tabular}{lcccccc}
\hline & CWI & ES & FR & CN & TUE & DE \\
\hline Adaptive refinement & $\checkmark$ & $\checkmark$ & & $\checkmark$ & & \\
Min grid size & $3.0 \mu \mathrm{m}$ & $3.9 \mu \mathrm{m}$ & $3.0 \mu \mathrm{m}$ & $2.0 \mu \mathrm{m}$ & NA & $4.2 \mu \mathrm{m}$ \\
Max grid size & & & & $8.0 \mu \mathrm{m}$ & & $5.0 \mu \mathrm{m}$ \\
Max $N_{\text {cells }}$ & $1.2 \times 10^{5}$ & $2.0 \times 10^{6}$ & $1.1 \times 10^{6}$ & $6.5 \times 10^{5}$ & $4.2 \times 10^{6}$ & $5.1 \times 10^{5}$ \\
Time step & dyn. & $1.0 \mathrm{ps}$ & dyn. & dyn. & dyn. & dyn. \\
CPU cores & 4 & 1 & 1 & 4 & 1 & 6 \\
Run time & $5 \mathrm{~min}$ & $20 \mathrm{~h}$ & $6 \mathrm{~h}$ & $18 \mathrm{~h}$ & $25 \mathrm{~h}$ & $15 \mathrm{~h}$ \\
\hline
\end{tabular}

Table 3. A summary of simulation settings for case 1, figure 7. We refer to the settings used for $\mathrm{CWI}_{0.8}, \mathrm{FR}_{0.8}$ and $\mathrm{DE}_{3.0}$ as 'higher' grid resolutions in the text. Please read section 4.2 for more details.

\begin{tabular}{lccccc}
\hline & $\mathrm{CWI}_{\{3.0,1.5,0.8\}}$ & $\mathrm{CWI}_{\mathrm{AMR}}$ & $\mathrm{FR}_{\{3.0,1.5,0.8\}}$ & $\mathrm{DE}_{\{5.0,4.0,3.0\}}$ & $\mathrm{DE}_{5.0}^{*}$ \\
\hline Adaptive refinement & & $\checkmark$ & & & \\
$\begin{array}{l}\text { Min grid size } \\
\text { Max grid size }\end{array}$ & $3.0,1.5,0.8 \mu \mathrm{m}$ & $3.0 \mu \mathrm{m}$ & $3.0,1.5,0.8 \mu \mathrm{m}$ & $4.2,3.2,2.2 \mu \mathrm{m}$ & $4.2 \mu \mathrm{m}$ \\
Time step & dyn. & dyn. & dyn. & $\begin{array}{c}\text { dyn. } \\
\text { dim }\end{array}$ & $\begin{array}{c}5.0 \mu \mathrm{m} \\
\text { dyn. }\end{array}$ \\
\hline
\end{tabular}

for $r>500 \mu \mathrm{m}$. These simulations took from several hours to three days.

4.2.2. ES. Figures 5, 6: AMR with a minimal grid size of $\Delta r=\Delta z=3.9 \mu \mathrm{m}$ (the coarsest grid size is four times larger) and a time step fixed at $1 \mathrm{ps}$ is employed. The maximum number of cells in the simulation domain is around
2013 184. The simulation took $20 \mathrm{~h}$ on an Intel Core i76700K CPU @ 4.00 GHz.

4.2.3. FR. Figures 5, 6 and figure $7\left(\mathrm{FR}_{3.0}\right): 4096$ cells along the $z$ direction (thus $\Delta z=3.05 \mu \mathrm{m}$ ) and 262 cells along the radial direction. $\Delta r=\Delta z$ remains constant until $600 \mu \mathrm{m}$ and is increased for $r>600 \mu \mathrm{m}$. The calculation took around $6 \mathrm{~h}$, 

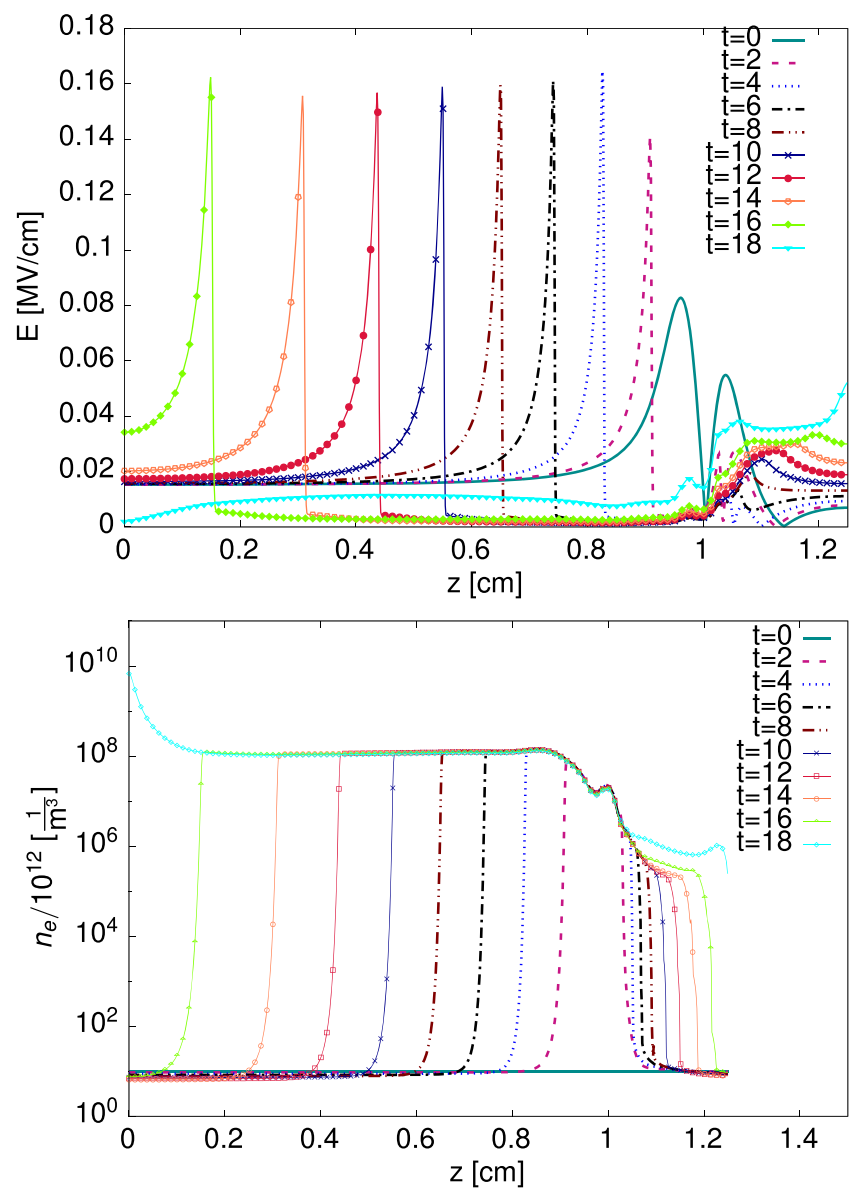

Figure 4. Case 1: the electric field strength (top) and electron density (bottom) on the streamer axis $r=0$ as a function of $z$ at different times (ns) using data from the CWI group. The details on the grid resolution are provided in section 4.2.1 and table 2 .

using one core on a desktop computer (Intel(R) Xeon(R) CPU X5272 $3.40 \mathrm{GHz})$.

Figure $7\left(\mathrm{FR}_{1.5}\right)$ has 8192 cells along the $z$ direction (thus $\Delta z=1.52 \mu \mathrm{m})$ and 400 cells along the radial direction. $\Delta r=\Delta z$ remains constant until $500 \mu \mathrm{m}$ and is increased for $r>500 \mu \mathrm{m}$. The calculation took around $32 \mathrm{~h}$ using one core on a desktop computer (Intel(R) Xeon(R) CPU X5272 $3.40 \mathrm{GHz}$ ).

Figure $7\left(\mathrm{FR}_{0.8}\right): 16384$ cells along the $z$ direction and 600 cells along the radial direction in which $\Delta z=0.76 \mu \mathrm{m}$. $\Delta r=0.96 \mu \mathrm{m}$ remains constant until $r=500 \mu \mathrm{m}$ and is increased for $r>500 \mu \mathrm{m}$. A parallelized code using the MPI libraries (the same specification as section 3.3 but using the direct R\&B SOR solver for elliptic equations) was run with 400 cores on the super computer EOS-CALMIP (612 computing nodes, each of them composed of 20 cores running at $2.8 \mathrm{Ghz}$ with $25 \mathrm{MB}$ of cache memory and sharing $64 \mathrm{~GB}$ of RAM). The simulation took around $25 \mathrm{~min}$.

4.2.4. $C N$. Figures 5, 6: AMR was used. The rectangular region $(0.7 \mathrm{~mm} \times 11.5 \mathrm{~mm})$ near the symmetry axis is constructed by triangular meshes with a maximum edge length
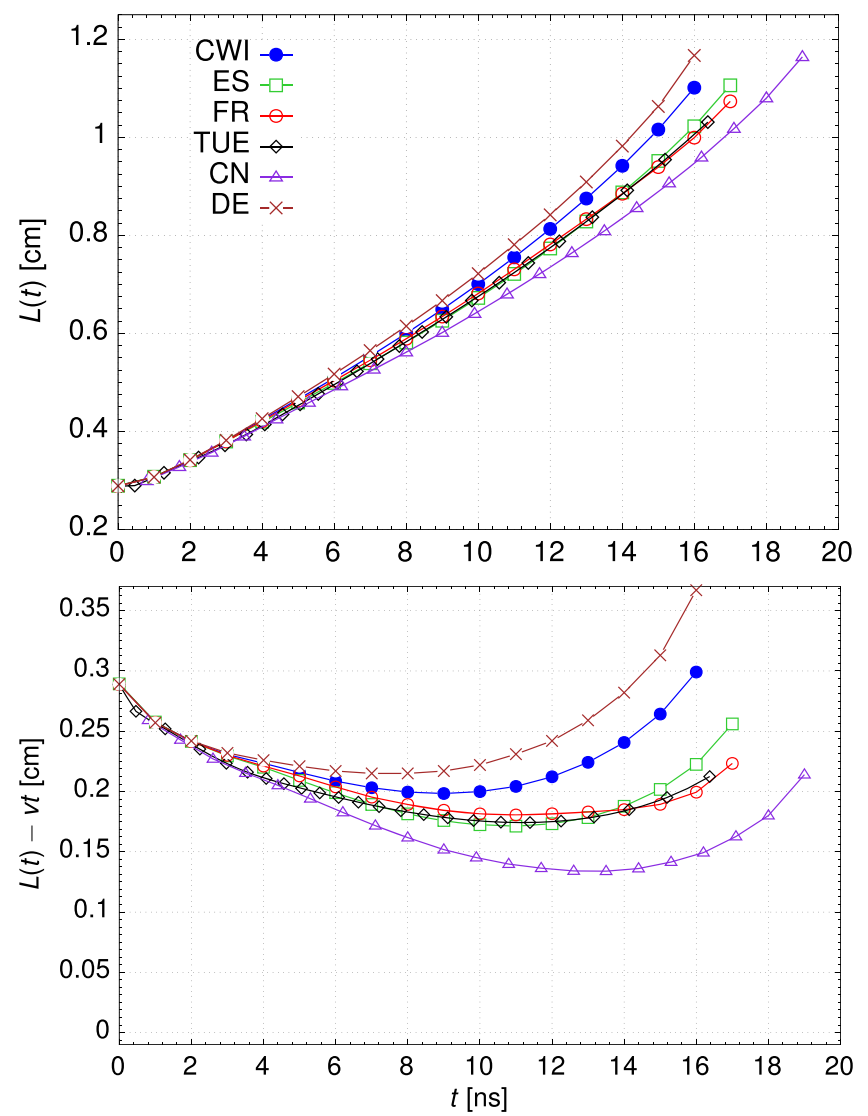

Figure 5. Case 1: the results for standard grid resolutions, see section 4.2 and table 2. Top: streamer length versus time; the streamer length is defined as $L(t)=1.25 \mathrm{~cm}-\mathrm{z}_{\max }(t)$, where $z_{\max }(t)$ is the location of the maximal electric field at time $t$. Bottom: to more clearly show the differences between the models, we subtract $v t$ from the streamer length, with $v=0.05 \mathrm{~cm} \mathrm{~ns}^{-1}$.

of $8 \mu \mathrm{m}$. In other regions a coarse mesh (maximum edge length $=93.8 \mu \mathrm{m}$ ) is employed. In the finer mesh region, the mesh is automatically refined according to the error indicator $\left(\sqrt{\left(\frac{\partial N_{e}}{\partial r}\right)^{2}+\left(\frac{\partial N_{e}}{\partial z}\right)^{2}}\right)$. The minimum mesh edge length is $2 \mu \mathrm{m}$. The initial number of cells is 389761 . As the streamer propagates, the maximum number of cells reaches 653994 . Time steps are automatically determined and the maximum step is set to $1.0 \times 10^{-11} \mathrm{~s}$. Finer initial mesh grids or time steps have not been further tested due to limited time and memory. The simulations were run on a desktop PC (Intel Xeon E31225@3.1 GHz, 8 GB RAM) and took around $18 \mathrm{~h}$.

4.2.5. TUE. Figures 5, 6: 8192 cells in the $z$ direction and 512 cells in the $r$ direction, concentrated around the axis of symmetry are used. The time step is limited to a maximum value of $1 \mathrm{ps}$. The linear systems that arise from this method are solved using the SuperLU solver [74]. The simulations were run using one core on a desktop PC (Intel i7-5820 K@3.3 GHz, 64 GB RAM). The simulation took around $25 \mathrm{~h}$.

4.2.6. $D E$. Hardware: all calculations were performed on a Linux compute node with Intel(R) Xeon(R) E5-2690 CPUs and 

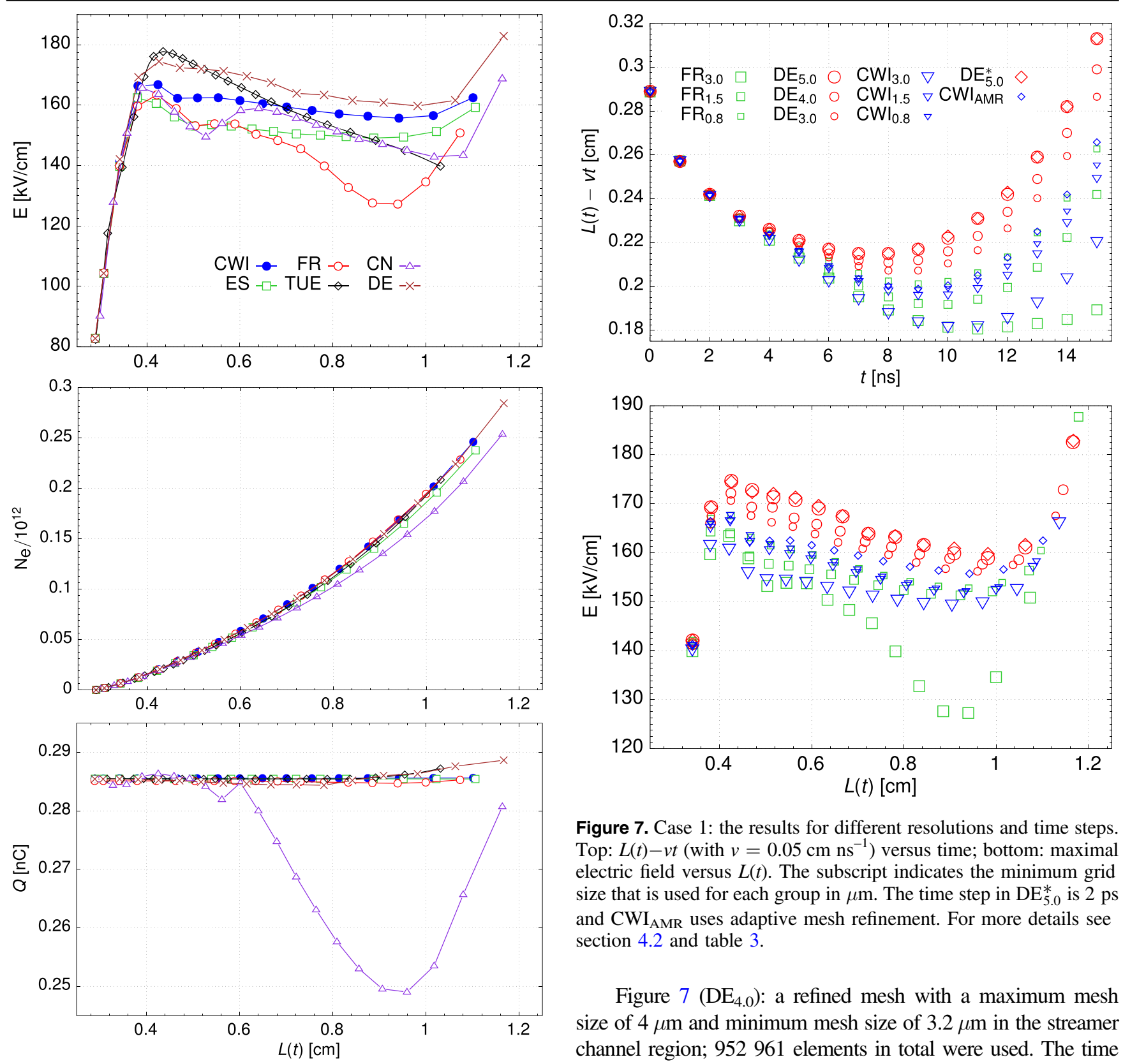

Figure 6. Case 1: the results for standard grid resolutions, see section 4.2 and table 2. From top to bottom: maximal electric field, total number of electrons and total charge in the system, all versus streamer length.

125 GB RAM. The automatic shared memory parallelization provided by COMSOL Multiphysics was used with six threads for each study.

Figures 5, 6 and figure $7\left(\mathrm{DE}_{5.0}\right)$ : in order to resolve the thin space charge region in front of the propagating streamer head, a locally refined triangular mesh with a maximum mesh size of $5 \mu \mathrm{m}$ and a minimum mesh size of $4.2 \mu \mathrm{m}$ in the streamer channel region was used for the numerical calculations. The complete mesh consists of 507615 elements. The time step size used by the time-implicit BDF solver was automatically adapted with an upper limit of $5 \mathrm{ps}$. The run time was $15 \mathrm{~h}$.

Figure 7. Case 1: the results for different resolutions and time steps. Top: $L(t)-v t$ (with $v=0.05 \mathrm{~cm} \mathrm{~ns}^{-1}$ ) versus time; bottom: maximal electric field versus $L(t)$. The subscript indicates the minimum grid size that is used for each group in $\mu \mathrm{m}$. The time step in $\mathrm{DE}_{5.0}^{*}$ is $2 \mathrm{ps}$ and $\mathrm{CWI}_{\mathrm{AMR}}$ uses adaptive mesh refinement. For more details see section 4.2 and table 3 .

Figure $7\left(\mathrm{DE}_{4.0}\right)$ : a refined mesh with a maximum mesh size of $4 \mu \mathrm{m}$ and minimum mesh size of $3.2 \mu \mathrm{m}$ in the streamer channel region; 952961 elements in total were used. The time step size used by the time implicit BDF solver was automatically adapted with an upper limit of $5 \mathrm{ps}$. The run time was $29 \mathrm{~h}$.

Figure $7\left(\mathrm{DE}_{3.0}\right)$ : a refined mesh with a maximum mesh size of $3 \mu \mathrm{m}$ and minimum mesh size of $2.2 \mu \mathrm{m}$ in the streamer channel region; 2246073 elements in total were used. The time step size used by the time implicit BDF solver was automatically adapted with an upper limit of $5 \mathrm{ps}$. The run time was $40 \mathrm{~h}$.

Figure $7\left(\mathrm{DE}_{5.0}{ }^{*}\right)$ : a refined triangular mesh with a maximum mesh size of $5 \mu \mathrm{m}$ in the streamer channel region was used for the numerical calculations. The complete mesh consists of 507615 elements. The time step size used by the time implicit BDF solver was automatically adapted with an upper limit of $2 \mathrm{ps}$. The run time was $31 \mathrm{~h}$.

\subsection{Results and discussion}

Below, we compare the results of the different models for the first test case. First, we present results with each group's 
standard grid resolution. Afterwards, we look into the convergence behavior when finer grids and smaller time steps are taken.

An example of the streamer evolution is shown in figure 3. The Gaussian seed of positive ions enhances the electric field, and within a couple of nanoseconds a positive streamer develops downwards. The streamer reaches the lower boundary within $20 \mathrm{~ns}$. To compare the models, we focus on the propagation stage and not on the dynamics after a conducting channel has formed between the electrodes. In figure 4 an example of electric field strength (top) and electron density (bottom) on the axis $(r=0)$, as a function of $z$ at different times is shown.

\subsubsection{Results with respective standard grid resolutions for} each group, see section 4.2. In figure 5 (top), the streamer length as a function of time is compared for the different models. We define the streamer length as $L(t)=L_{z}-z_{\max }(t)$, where $z_{\max }(t)$ is the location of the maximal electric field at time $t$ and $L_{z}=1.25 \mathrm{~cm}$ is the domain size. To more clearly show the differences between the models, figure 5 (bottom) shows $L(t)-v t$, where $v=0.05 \mathrm{~cm} \mathrm{~ns}^{-1}$. The differences between the models grow over time, and at $t=16 \mathrm{~ns}$ there is about $2 \mathrm{~mm}$ difference between $\mathrm{DE}$ (the fastest) and $\mathrm{CN}$ (the slowest).

The streamer position over time is sensitive to small numerical differences between the models. A difference in position grows over time, because a streamer that has propagated further also has a higher velocity, which can be seen from the increasing slopes in figure 5. The maximal electric field, the total number of electrons and the total charge in the system are shown in figure 6 . These quantities are shown versus the streamer length, so that the differences in the physical predictions of the models can more clearly be seen. Note that agreement in figure 6 does not imply agreement in streamer position or velocity.

Figure 6 (top) shows that the maximal electric field first rises rapidly (indicating that a streamer has formed), and then it slowly decreases as the streamer propagates across the gap. When the streamer gets close to the other boundary, an increase is again visible. The predictions of the CWI, ES, CN and DE models are within about $20 \mathrm{kV} \mathrm{cm}^{-1}$, whereas the FR results start to deviate about halfway through the gap. This difference disappears when a finer mesh is used, as will be shown in section 4.3.2.

The total number of electrons versus streamer length shows quite good agreement between the models (figure 6 (middle)). This implies that the 'line' electron density along the streamer (obtained by integrating over the radial direction) is similar for the models, and that it is not as sensitive to the numerical implementation as the velocity or the maximal electric field.

The total charge $Q$ in the system is shown in figure 6 (bottom). A positive charge of about $0.285 \mathrm{C}$ is created by the initial seed of positive ions. The streamer discharge itself should contain an equal number of electrons and ions, so that $Q$ should be conserved except for small fluxes through the domain boundaries. The differences between the models indicate that there are also numerical errors in charge conservation, in particular for finite-element-based codes. The finite volume methods should in principle conserve charge, as the fluxes are always between neighboring cells. For the CWI model, small errors can come from the adaptive mesh refinement technique, which here used a nonconservative interpolation procedure.

Finite element methods do not automatically ensure conservation, which could explain the deviations observed in figure 6 (bottom). Another reason could be the logarithmic representation of the drift-diffusion equations by $\mathrm{CN}$, as described in section 3. Furthermore, a feature called 'source stabilization' is used in the $\mathrm{CN}$ model, which adds a source term $R_{e}=N_{A} \exp \left(-\zeta \ln \left(n_{e}\right)\right)$ to the electron density, where $\zeta=0.4$ is a user-defined tuning parameter. Since this term is only added to the electrons, net charge is produced (it could also be added to the ions to avoid this).

4.3.2. Results with higher grid resolutions. Since the compared models solve the same equations, differences between them should go to zero when $\Delta x$ and $\Delta t$ go to zero. Here we investigate whether that is indeed the case, with results from the DE, FR and CWI groups. The other participating groups did not run simulations on finer grids due to computational and/or time restrictions.

Figure 7 shows the streamer length and the maximal electric field for the FR, DE and CWI models with different grid resolutions. Smaller symbols indicate smaller grid cells, and the subscripts indicate the mesh spacing in $\mu \mathrm{m}$. For the FR and CWI results, a uniformly refined mesh up to $r=0.5 \mathrm{~mm}$ or $r=0.6 \mathrm{~mm}$ is used. Surprisingly, finer grids for the DE model lead to a slower streamer with a lower maximal electric field, whereas the opposite trend is present in the CWI and FR results. For the finest grids, the maximal difference in streamer length at $t=15 \mathrm{~ns}$ is about $0.3 \mathrm{~mm}$, and it occurs between the $\mathrm{CWI}_{0.8}$ and $\mathrm{DE}_{3.0}$ results. The difference between the $\mathrm{CWI}_{0.8}$ and $\mathrm{FR}_{0.8}$ streamer length is much smaller, namely less than $0.04 \mathrm{~mm}$. From the trends shown in this figure, the DE results are expected to get closer to the CWI and FR results if a similarly fine mesh is used.

The models also appear to converge in their maximal electric field, with the differences between the $\mathrm{CWI}_{0.8}$ and $\mathrm{FR}_{0.8}$ uniform grid results again being the smallest. The $\mathrm{CWI}_{3.0}$ and $\mathrm{FR}_{3.0}$ results look quite different, however, as the $\mathrm{FR}_{3.0}$ results show a minimum in electric field at $L=0.9 \mathrm{~cm}$, which disappears for finer grids.

For comparison, the CWI results with an adaptive mesh are also shown in figure 7 . For the AMR results, the finest grid corresponds to the $\mathrm{CWI}_{3.0}$ case, but they lie closer to the $\mathrm{CWI}_{0.8}$ results. The adaptive refinement perhaps slightly enhances growth in the forward direction (as compared to radial expansion), which enhances the streamer's velocity and maximal electric field. The $\mathrm{DE}_{5.0}^{*}$ case uses a smaller maximal time step ( $2 \mathrm{ps}$ ) than the $\mathrm{DE}_{5.0}$ case $(5 \mathrm{ps})$, but there are no noticeable differences between them.

In conclusion, we find reasonably good agreement between the models if sufficiently small time steps and grid spacing are used. Even though the first test case was designed 
Table 4. A summary of the simulation settings for case 2 , figures $8-10$. We refer to this setting as 'standard' grid resolution for case 2 in the text. Please read section 5.2 for more details. Note that for DE and $\mathrm{CN}$, the min and max grid size are specified in the narrow rectangular region where the streamer propagates and not in the whole simulation domain.

\begin{tabular}{lccccc}
\hline & CWI & ES & FR & CN & DE \\
\hline Adaptive refinement & $\checkmark$ & $\checkmark$ & & $\checkmark$ & \\
Min grid size & $3.0 \mu \mathrm{m}$ & $3.9 \mu \mathrm{m}$ & $1.52 \mu \mathrm{m}$ & $2.0 \mu \mathrm{m}$ & $2.1 \mu \mathrm{m}$ \\
Max grid size & & & & $8.0 \mu \mathrm{m}$ & $3.0 \mu \mathrm{m}$ \\
Max $N_{\text {cells }}$ & $5.7 \times 10^{4}$ & $2.0 \times 10^{6}$ & $3.3 \times 10^{6}$ & $6.6 \times 10^{5}$ & $1.3 \times 10^{6}$ \\
Time step & dyn. & $1.0 \mathrm{ps}$ & dyn. & dyn. & dyn. \\
CPU cores & 4 & 1 & 1 & 4 & 6 \\
Run time & $5 \mathrm{~min}$ & $36 \mathrm{~h}$ & $45 \mathrm{~h}$ & $18 \mathrm{~h}$ & $61 \mathrm{~h}$ \\
\hline
\end{tabular}

to be computationally relatively easy, fine grids (and therefore costly simulations) are required to get close to numerical convergence.

\section{Second test case: low background ionization}

\subsection{Description}

The second test case is identical to the first one, except for the use of a $10^{4}$ times lower background ionization level of $n_{i}=n_{e}=10^{9} \mathrm{~m}^{-3}$. A lower background density typically leads to a positive streamer with a smaller radius, a higher electric field at its tip, and larger density gradients. For this reason, the second test case is computationally and numerically more challenging than the first one.

\subsection{Simulation information}

A summary of the simulation settings is provided in tables 4 and 5 .

5.2.1. CWI. Figures 8-11: an AMR with a minimum grid size of $\Delta r=\Delta z=3.05 \mu \mathrm{m}$ is used. The refinement criterion is the following: refine if $\alpha(1.2 E) \Delta x>1.0$ and de-refine if $\alpha(1.2 E) \Delta x<1.0$, where $\alpha(E)$ is the field-dependent ionization coefficient, $E$ is the electric field strength in $\mathrm{V}$ $\mathrm{m}^{-1}$ and $\Delta x$ is the cell spacing. The maximum number of cells is $5.7 \cdot 10^{4}$. The computer specification is the same as for case 1 in section 4.2.1. The simulation took around $5 \mathrm{~min}$.

Figure 11: a fixed grid as for case 1 (section 4.2.1). The simulations took between several hours and 2 to 3 days.

5.2.2. ES. Figures 8, 9: the grid resolution and the computer specification are the same as in case 1 (section 4.2.2). The simulation took $36 \mathrm{~h}$.

5.2.3. FR. Figures 8,9 and $11\left(\mathrm{FR}_{1.5}\right): 8192$ cells along the $z$ direction and 400 cells along the radial direction are used, in which the grid size $\Delta z=\Delta r=1.52 \mu \mathrm{m}$ remains constant until $r=500 \mu \mathrm{m}$ and is increased for $r>500 \mu \mathrm{m}$. The calculation took around $45 \mathrm{~h}$ using one core on a desktop computer (Intel(R) Xeon(R) CPU X5272 3.40 GHz).

Figures $12,11\left(\mathrm{FR}_{0.8}\right)$ : 16384 cells along the $z$ direction and 600 cells along the radial direction in which
Table 5. A summary of the simulation settings for case 2, figure 11 . We refer to the settings used for $\mathrm{CWI}_{0.8}, \mathrm{FR}_{0.8}$ as the 'higher' grid resolutions for case 2 in the text. Please read section 5.2 for more details.

\begin{tabular}{lccc}
\hline & $\mathrm{CWI}_{\{3.0,1.5,0.8\}}$ & $\mathrm{CWI}_{\mathrm{AMR}}$ & $\mathrm{FR}_{\{3.0,1.5,0.8\}}$ \\
\hline $\begin{array}{l}\text { Adaptive } \\
\text { refinement }\end{array}$ & $\checkmark$ & \\
$\begin{array}{l}\text { Min grid size } \\
\text { Time step }\end{array}$ & $\begin{array}{c}3.0,1.5,0.8 \mu \mathrm{m} \\
\text { dyn. }\end{array}$ & $\begin{array}{c}3.0 \mu \mathrm{m} \\
\text { dyn. }\end{array}$ & $\begin{array}{c}3.0,1.5,0.8 \mu \mathrm{m} \\
\text { dyn. }\end{array}$ \\
\hline
\end{tabular}

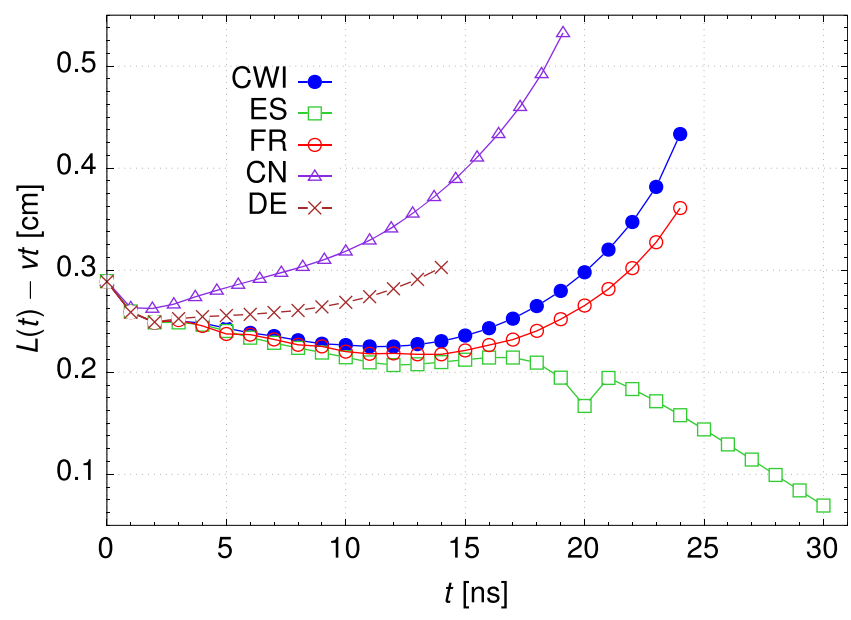

Figure 8. Case 2: a comparison of the streamer lengths for standard grid resolutions, see section 5.2 and table 4 . To clearly show the differences $L(t)-v t$ is shown, with $v=0.03 \mathrm{~cm} \mathrm{~ns}^{-1}$.

$\Delta z=0.76 \mu \mathrm{m} . \quad \Delta r=0.96 \mu \mathrm{m} \quad$ remains constant until $r=500 \mu \mathrm{m}$ and is increased for $r>500 \mu \mathrm{m}$. A parallelized code using the MPI libraries (the same specification as section 3.3 but using the direct R\&B SOR solver for elliptic equations) was run with 400 cores on the super computer EOS-CALMIP (612 computing nodes, each of them being composed of 20 cores running at $2.8 \mathrm{Ghz}$ with $25 \mathrm{MB}$ of cache memory and sharing 64 GB of RAM). The simulation takes around $36 \mathrm{~min}$.

5.2.4. $C N$. Figure 8: the grid resolution and the computer specification is the same as case 1 in section 4.2.4. The initial number of cells is 389761 . As the streamer propagates, the 

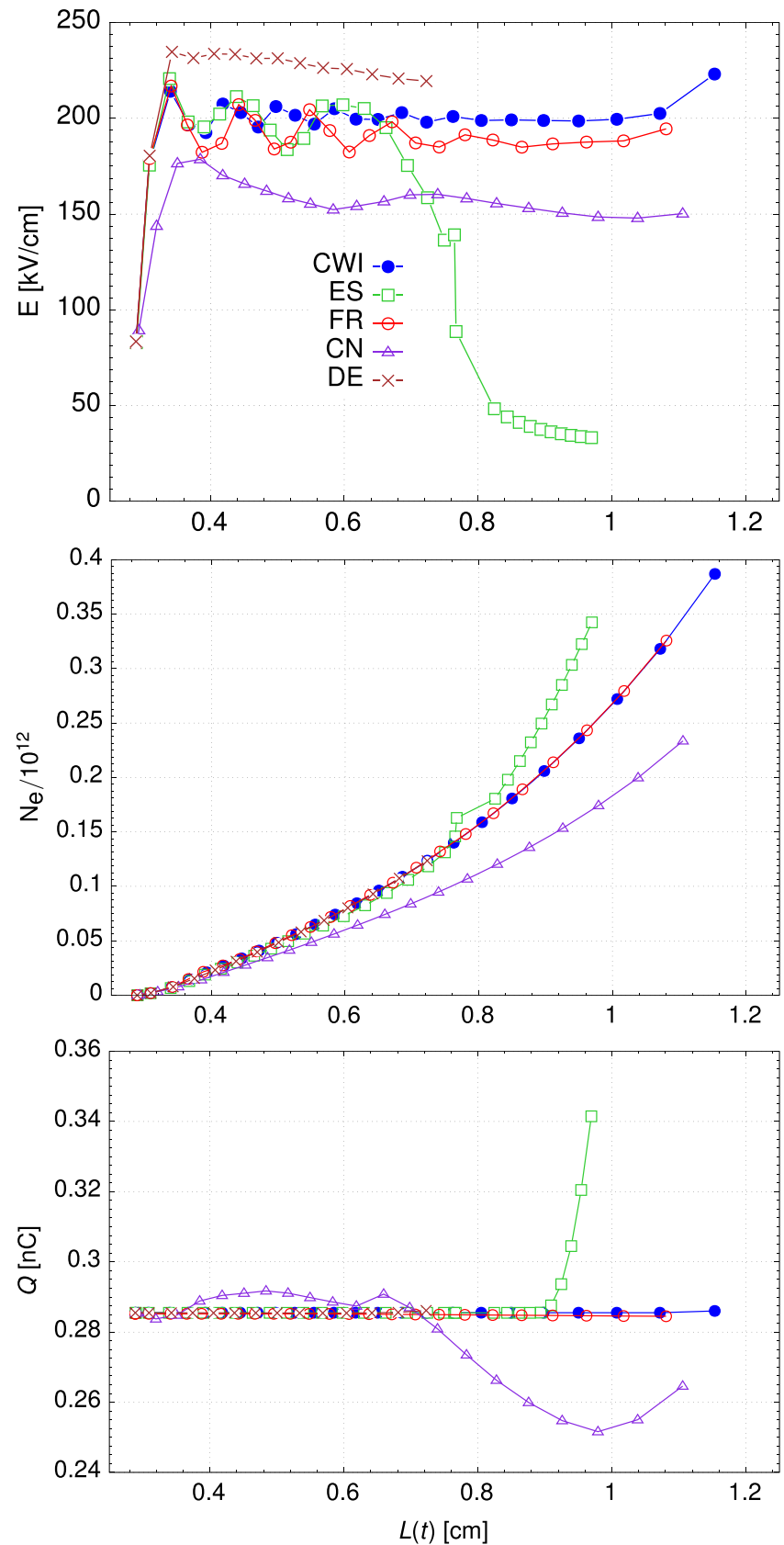

Figure 9. Case 2: the results for standard grid resolutions, see section 5.2 and table 4 . From top to bottom: the maximum electric field, the total number of electrons and the total charge in the system, all versus streamer length. For the ES model the maximal on-axis electric field is shown, which decreases after the streamer branches.

maximum number of cells reaches 658395 . The simulation took $18 \mathrm{~h}$.

5.2.5. $D E$. Figure 8: a locally refined triangular mesh with a maximum mesh size of $3 \mu \mathrm{m}$ in the streamer channel region was used for the numerical calculations. The complete mesh consists of 1297826 elements. The time step size used by the time implicit BDF solver was automatically adapted with an

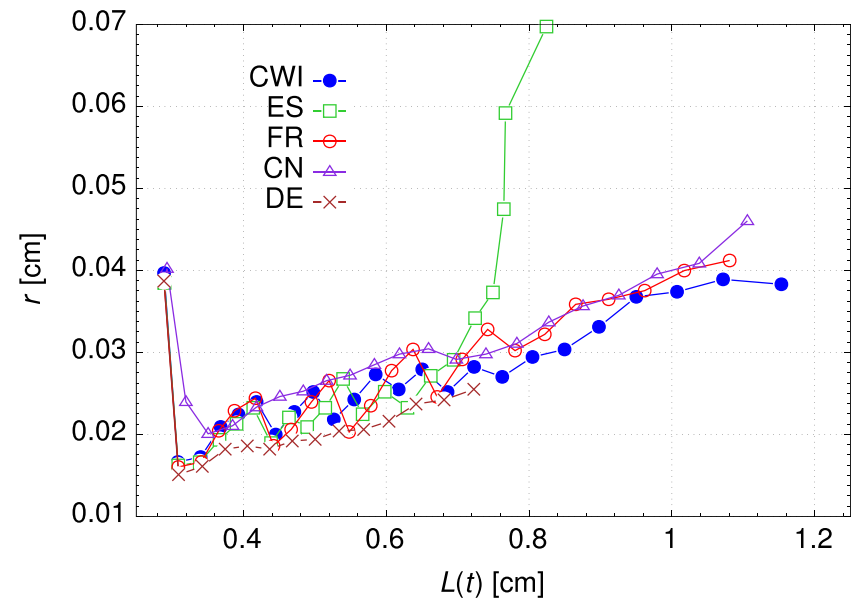

Figure 10. Case 2: the results for standard grid resolutions, see section 5.2. The radius of the streamer head versus streamer length is shown. The simulation information is provided in section 5.2 and table 4 .
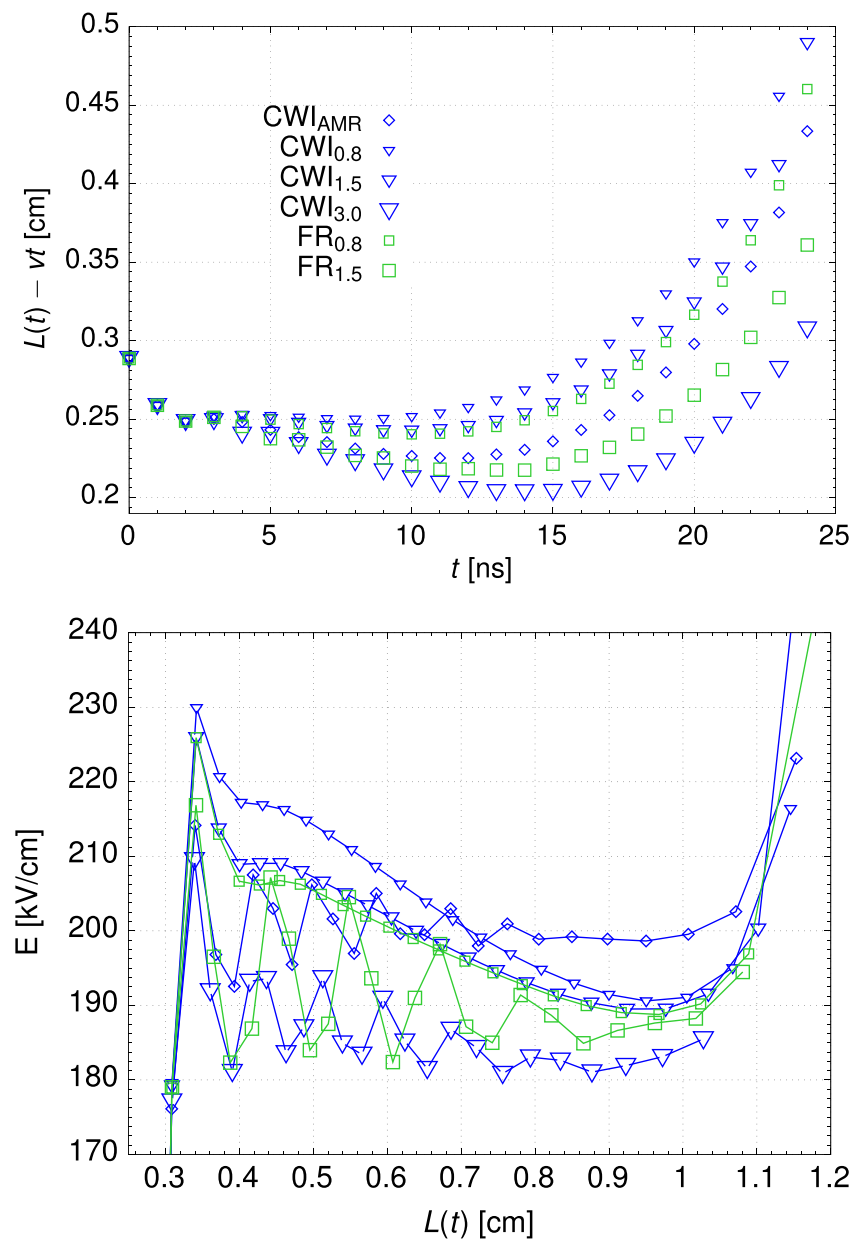

Figure 11. Case 2: with different grid resolutions for CWI and FR. Top: $L(t)-v t$ (with $v=0.03 \mathrm{~cm} \mathrm{~ns}^{-1}$ ) versus time; bottom: maximum electric field versus $L(t)$. The subscript indicates the minimum grid size that is used for each group in $\mu \mathrm{m}$. For more details see section 5.2. In $\mathrm{CWI}_{\mathrm{AMR}}, \mathrm{CWI}_{3.0}$ and $\mathrm{FR}_{1.5}$ oscillations in the electric field are present, see also figure 12. For finer grids the oscillations disappear. 


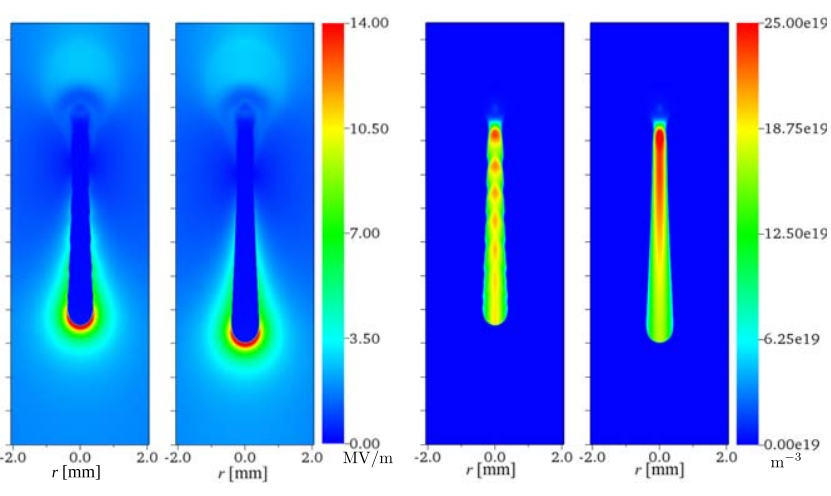

Figure 12. Case 2: the electric field strength $|\mathbf{E}|$ (left) and electron density (right) at $t=20 \mathrm{~ns}$. Per plot, the left figure shows the CWI AMR results (with oscillations) and the right figure shows the CWI results with a uniform grid of $0.8 \mu \mathrm{m}$ (without oscillations). Similar observations were present in the FR results; they disappear for sufficiently fine grids. See table 5 for information about the grid resolutions.

upper limit of $2 \mathrm{ps}$. The computer specifications are the same as case 1 in section 4.2.6, the simulation took $61 \mathrm{~h}$.

\subsection{Results and discussion}

We first present the results for each group's standard grid resolution. The evolution of the streamer length of the propagating streamer is plotted in figure 8 . After $14 \mathrm{~ns}$ the DE simulations crash due to a numerical instability, and the streamer branches for the ES simulations at a later time, confirming that case 2 is computationally more challenging than case 1 . The other models did not experience such instabilities.

Compared to case 1 , it takes longer for the streamer to bridge the gap. The $\mathrm{CN}$ streamer is the fastest, unlike for case 1. The DE results also show a relatively fast streamer. The other codes show similar behavior up to $t=16 \mathrm{~ns}$, after which streamer branching occurs in the ES model, and it starts to deviate. We remark that true streamer branching cannot be simulated in an axisymmetric model.

The maximal electric field, the total number of electrons and the total charge in the system are shown in figure 9. $\mathrm{CN}$ has the lowest maximal electric field, and its predictions for the total number of electrons and total charge deviates from that of the other models. This probably indicates that a finer grid and/or a smaller time step are required to simulate case 2 with the $\mathrm{CN}$ model. The results of CWI and FR agree well, and they are close to the ES results up to $L=0.7 \mathrm{~cm}$. The DE results up to $L=0.7 \mathrm{~cm}$ show a slightly higher electric field than the CWI, FR and ES results, but the models show good agreement in terms of total electron number and charge conservation.

Both the CWI and FR results include oscillations in the electric field and electron density profile. Figure 10 shows the radius of the streamer head (defined as the radius where the radial component of the electric field is maximal) versus the streamer length in which one can perceive the oscillations in radius. These oscillations disappear when a finer grid is used, as discussed below. Perhaps the branching observed with the ES model is a result of similar, but stronger oscillations. However, the $\mathrm{CN}$ and DE COMSOL models, which both perform implicit time stepping, did not exhibit these oscillations.

5.3.1. Results with higher grid resolutions. For the CWI and FR models, additional simulations at higher grid resolutions were performed, using a uniformly refined mesh of up to $r=0.5 \mathrm{~mm}$. The resulting streamer length and maximal electric field are shown in figure 11. The oscillations in maximal electric field (and electron density) disappear for finer grids, as shown in figure 12.

Regarding streamer length, the difference between the $\mathrm{CWI}_{3.0}$ and $\mathrm{CWI}_{1.5}$ results is much larger than the difference between $\mathrm{CWI}_{1.5}$ and $\mathrm{CWI}_{0.8}$. This could indicate that $\mathrm{CWI}_{0.8}$ is close to numerical convergence, but surprisingly $\mathrm{FR}_{0.8}$ agrees better with $\mathrm{CWI}_{1.5}$, and in the maximal electric field as well. The difference in position between $\mathrm{CWI}_{0.8}$ and $\mathrm{FR}_{0.8}$ at $t=23 \mathrm{~ns}$ is about $0.6 \mathrm{~mm}$, which is considerably larger than the difference of $0.04 \mathrm{~mm}$ observed for case 1 . As for case 1, the CWI AMR results show a higher streamer velocity than the $\mathrm{CWI}_{3.0}$ results, even though they use the same finest grid.

In conclusion, we can get a reasonable agreement between the CWI and FR models for sufficiently fine grids, but with the same numerical resolution the agreement is worse than for case 1 . The lower background electron density makes case 2 highly challenging. Oscillations, instabilities and streamer branching can occur due to the higher electric fields at the streamer tip and the steeper gradients in electron density.

\section{Third test case: including photoionization}

\subsection{Description}

The third test case is the same as the second one with an initial electron and ion density of $10^{9} \mathrm{~m}^{-3}$, except that photoionization is now included. All groups implemented photoionization based on Zhelenznyak's model (see section 2.3), using the Helmholtz approach to solve the photoionization integral. For more details see appendix A. Each group uses either Luque et al's [66] or Bourdon et al's [55] parametrization. The subscript $L$ indicates parameters from Luque et $a l$, and subscript $B_{2}\left(B_{3}\right)$ represents the two (three)-term Bourdon et al parameters. When there is no subscript, threeterm Bourdon et al parameters are used.

\subsection{Simulation information}

In the following, details of the simulation (grid refinement, computer specification and simulation time) are discussed. A summary of simulation settings is provided in table 6 .

6.2.1. CWI. Figures 13-17: an AMR with a minimum grid size of $\Delta r=\Delta z=3.05 \mu \mathrm{m}$ is used. The maximum number of cells in the simulation domain is 74560 for $L, 75904$ for $B_{2}$, and 76480 for $B_{3}$. The computer specification is the same 
Table 6. A summary of simulation settings for case 3, figures 13-15. Bourdon's three-term parameters are used. Note that for DE and CN, the min and max grid size are specified in the narrow rectangular region where the streamer propagates and not in the whole simulation domain.

\begin{tabular}{lccccc}
\hline & CWI & ES & FR & CN & DE \\
\hline Adaptive refinement & $\checkmark$ & $\checkmark$ & & $\checkmark$ & \\
Min grid size & $3.0 \mu \mathrm{m}$ & $3.9 \mu \mathrm{m}$ & $6.0 \mu \mathrm{m}$ & $2.0 \mu \mathrm{m}$ & $4.1 \mu \mathrm{m}$ \\
Max grid size & & & & $8.0 \mu \mathrm{m}$ & $5.0 \mu \mathrm{m}$ \\
Max $N_{\text {cells }}$ & $7.6 \times 10^{4}$ & $2.0 \times 10^{6}$ & $2.9 \times 10^{5}$ & $7.2 \times 10^{5}$ & $7.6 \times 10^{5}$ \\
Time step & dyn. & $1.0 \mathrm{ps}$ & dyn. & dyn. & dyn. \\
CPU cores & 4 & 1 & 1 & 4 & 6 \\
Run time & $<5$ min & $72 \mathrm{~h}$ & 34 min & $26 \mathrm{~h}$ & $42 \mathrm{~h}$ \\
& & & & & \\
\hline
\end{tabular}

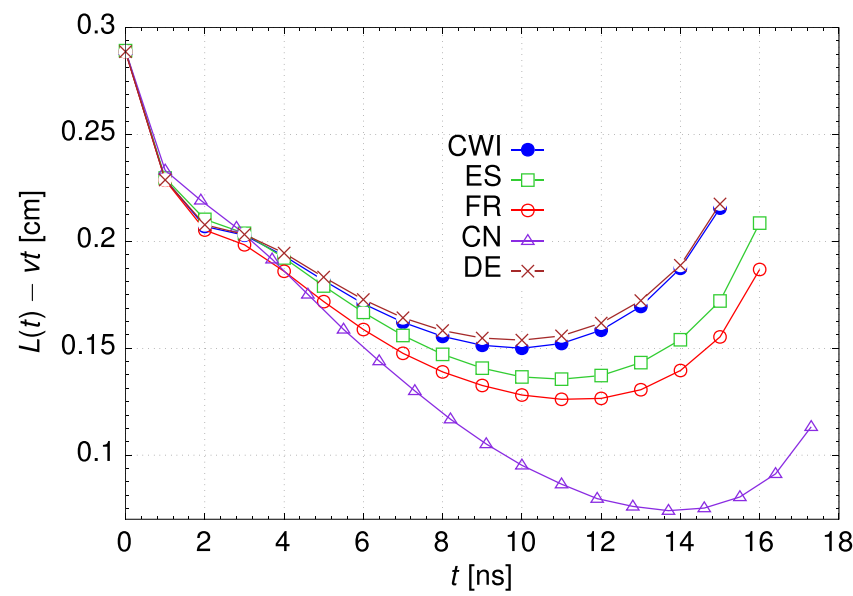

Figure 13. Case 3: the results with Bourdon's [55] three-term parameters, see section 6 and table 6 . To more clearly show the differences between the models we show $L(t)-v t$, where $L(t)$ is the streamer length and $v=0.06 \mathrm{~cm} \mathrm{~ns}^{-1}$.

as for case 1 in section 4.2.1. The refinement criterion is the same as case 2 in section 5.2.1. The simulations took around 2-3 min.

6.2.2. ES. Figures 13-15: the grid resolution and computer specification are the same as for case 1 in section 4.2.2. The calculation took around 3 days.

6.2.3. FR. Figures $13-15\left(\mathrm{FR}_{6.0_{L, B}, B_{3}}\right): 2047$ cells along the $z$ direction and 141 cells along the radial direction are used. In this case the simulations are first order in time and it took around $34 \mathrm{~min}$.

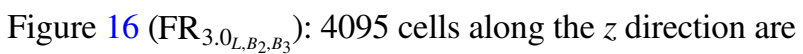
used. In this case the simulations are first order in time and it took around $4 \mathrm{~h}$.

6.2.4. CN. Figures 13-15: the grid and computer specifications are the same as for case 1, section 4.2.4 is used. The initial number of cells is 389 761. As the streamer propagates, the maximum number of cells reaches 719773 ; the simulation took around $26 \mathrm{~h}$.

6.2.5. DE. Figures 13, 14: a locally refined triangular mesh with a maximum mesh size of $5 \mu \mathrm{m}$ in the streamer channel region was used for the numerical calculations. The complete
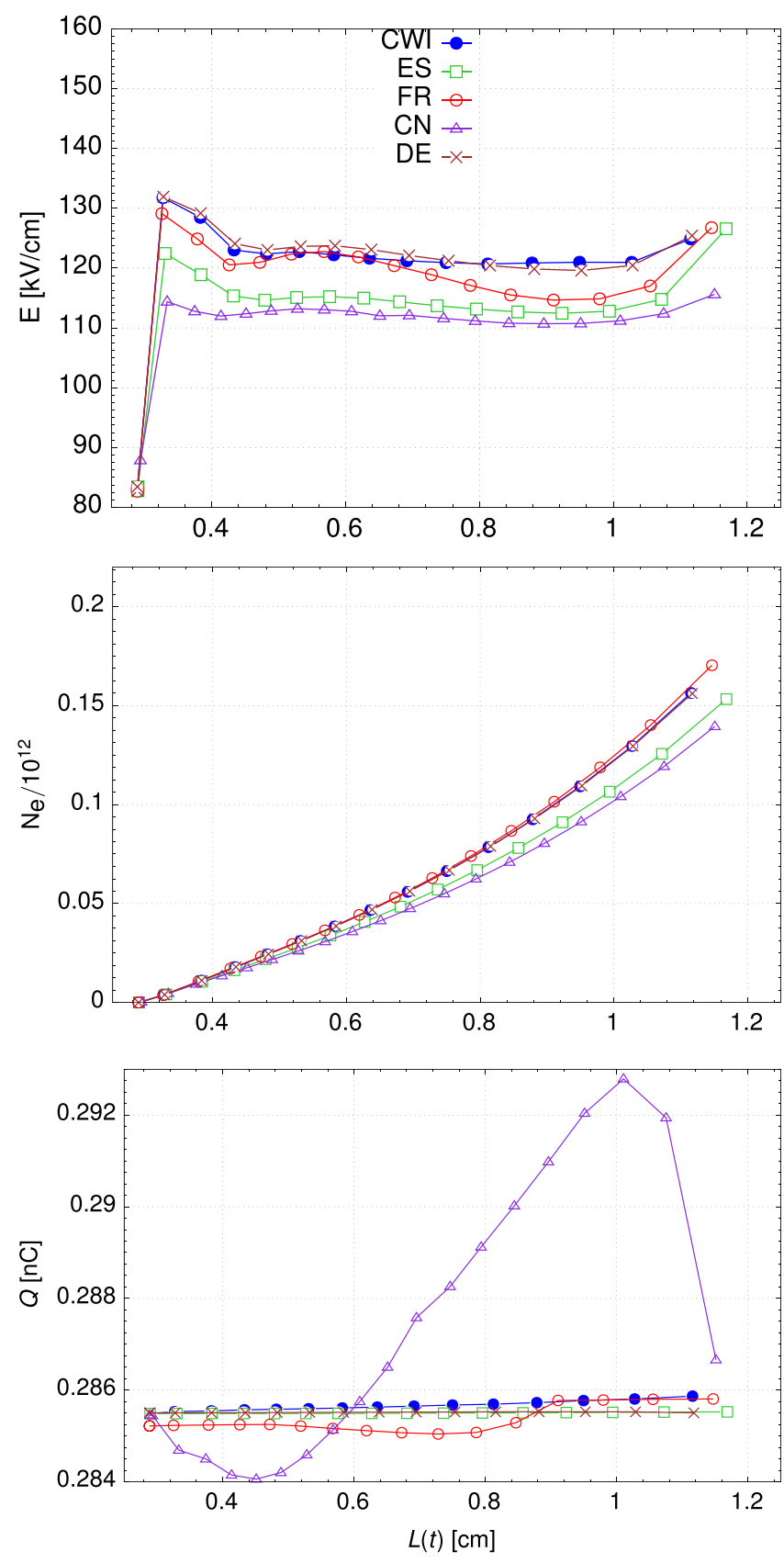

Figure 14. Case 3: the results with Bourdon's [55] three-term parameters, see section 6 and table 6. From top to bottom: the maximum electric field, the total number of electrons and total charge, all versus streamer length. 


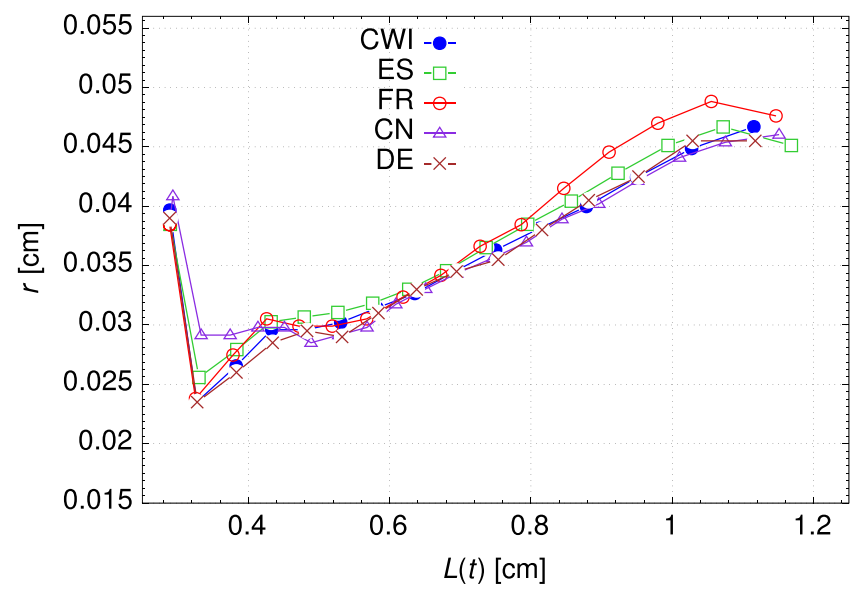

Figure 15. Case 3: the results with Bourdon's [55] three-term parameters, see section 6 and table 6 . The radius of the streamer head versus streamer length is plotted.
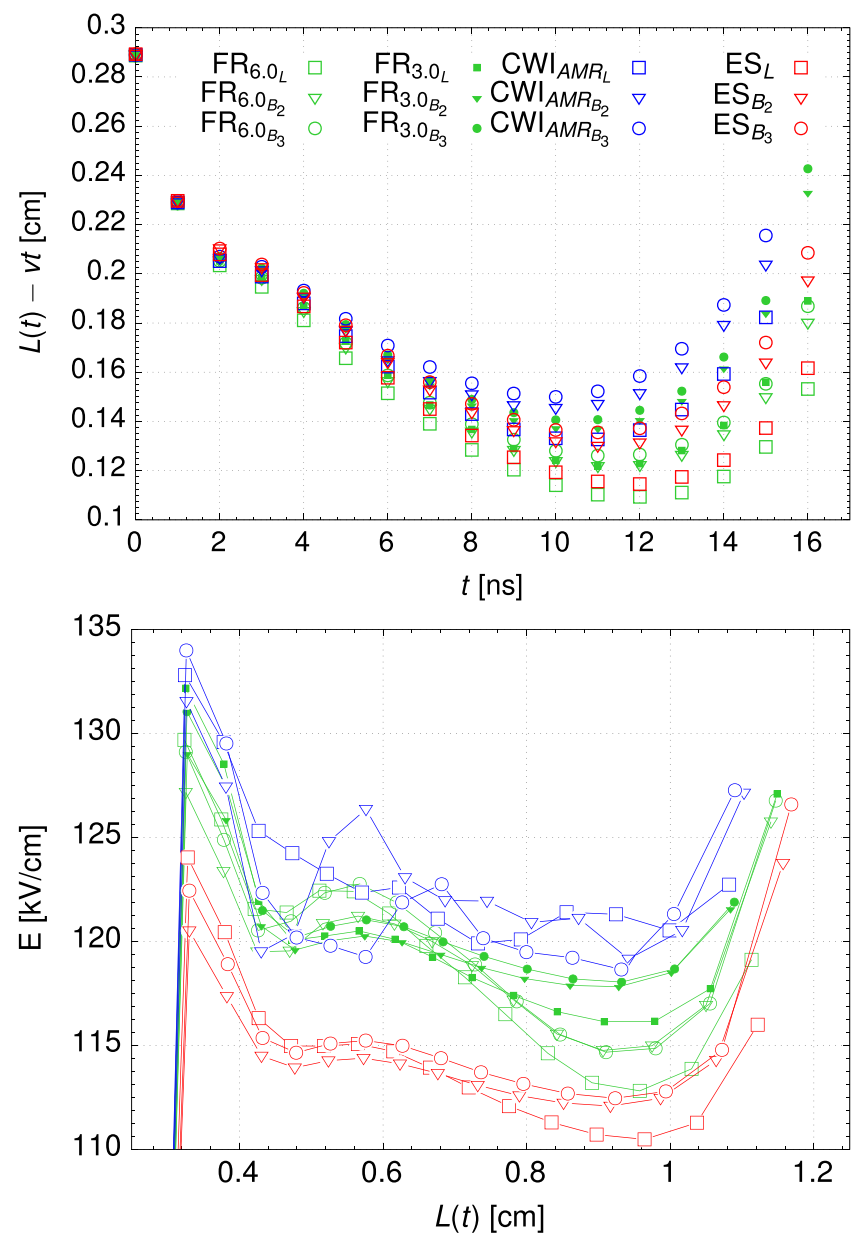

Figure 16. Case 3: the results with different photoionization parameters $\left(L, B_{2}\right.$ and $\left.B_{3}\right)$. Top: $L(t)-v t$ (with $v=0.06 \mathrm{~cm} \mathrm{~ns}^{-1}$ ) versus time; bottom: maximum electric field versus $L(t)$. The subscript indicates the minimum grid size that is used in $\mu \mathrm{m}$. The subscripts $L, B_{2}$ and $B_{3}$ represent Luque's [66], Bourdon's two-term and Bourdon's three-term [55] fitting, respectively.
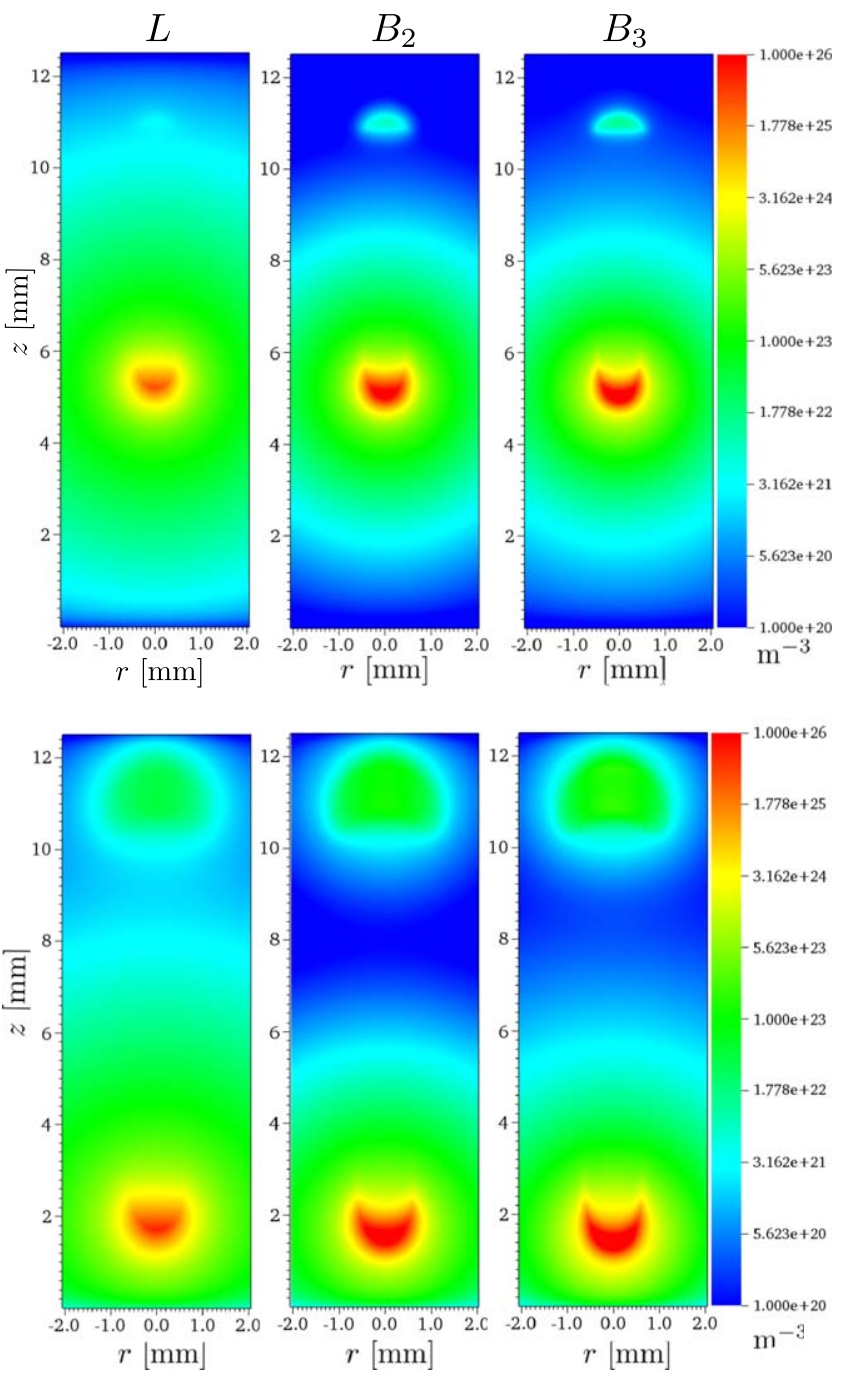

Figure 17. Case 3: a profile of photoelectrons produced using data from CWI. Top: $t=10 \mathrm{~ns}$, bottom: $t=15 \mathrm{~ns} ; L, B_{2}$ and $B_{3}$ stands for Luque's [66], Bourdon's two-term and Bourdon's three-term [55], respectively.

mesh consists of 764198 elements. The time step size used by the time implicit BDF solver was automatically adapted with an upper limit of $5 \mathrm{ps}$. The computer specifications are the same as case 1 in section 4.2.6; the simulation took $42 \mathrm{~h}$.

\subsection{Results and discussion}

We now compare the results of different groups. First we present the results with Bourdon's three-term parameters $\left(B_{3}\right)$. Then, we investigate how the results vary when Luque's $(L)$ or Bourdon's two-term parameters $\left(B_{2}\right)$ are used.

6.3.1. Results with $B_{3}$ parameters. In figure 13 the streamer lengths as a function of time are compared. Similar observations as for the first test case can be made: the difference in position between the models grows over time, 
and at $t=15 \mathrm{~ns}$ there is about $2 \mathrm{~mm}$ difference between CWI (or DE) and CN (the slowest). CWI and DE agree quite well, as do ES and FR.

The maximal electric field, the total number of electrons and the total charge in the system are shown in figure 14. As in section 4 these quantities are shown versus the streamer length in order to see the differences in the physical prediction of the models more clearly. Compared to case 1, the maximal electric field is lower, and it varies less as the streamer propagates through the gap. The predictions of all participants are within about 15 to $20 \mathrm{kV} \mathrm{cm}^{-1}$. The maximal electric field of CWI and DE coincides quite well. The total number of electrons versus streamer length shows larger differences between the groups than for cases 1 or 2 , but the reason behind this is unclear. The total charge $Q$ in the system is shown in the lowest panel of figure 14. The DE and ES models have the smallest variation in total charge, followed by the FR and CWI models. For the CWI results, nonconservation can come from the AMR procedure, which here used nonconservative interpolation. The $\mathrm{CN}$ results show the largest deviation, as for the previous test cases, see the discussion in section 4.3. The radius of the streamer head is shown in figure 15. All the models give a similar radius until the streamer length reaches around $0.8 \mathrm{~cm}$. For $L>0.8 \mathrm{~cm}$, the radius by the FR model starts deviating by around $5 \times 10^{-3} \mathrm{~cm}$. Overall, one finds reasonable agreement between all the models.

6.3.2. Results with $L, B_{2}$ and $B_{3}$ parameters. Here we investigate how the results vary when different coefficients for the Helmholtz approximation are used. In figure 16 the streamer length and the maximal electric field for FR, CWI and ES are shown. Different symbols show different photoionization parameters. Luque's $(L)$ are shown by squares, Bourdon's two-term $\left(B_{2}\right)$ by down triangles, and Bourdon's three-term $\left(B_{3}\right)$ by open circles. Filled points indicate the result of FR with a finer grid resolution. The results show that with $B_{3}$ the steamer is the fastest and with $L$ it is the slowest. The differences between groups using the same photoionization parameters are (at least partially) caused by discretization errors. These numerical differences seem to be more important than the choice of photoionization parameters. CWI uses a finer grid than FR and ES, and predicts a faster streamer. When the mesh size is decreased from $6 \mu \mathrm{m}$ to $3 \mu \mathrm{m}$ in FR their results get close to CWI.

Figure 17 shows the profile of photoelectrons $\left(\mathrm{m}^{-3}\right)$ for CWI using $L, B_{2}$ and $B_{3}$ at time $t=10 \mathrm{~ns}$ (top) and $t=15 \mathrm{~ns}$ (bottom). The results obtained with different photoionization parameters are very similar.

\section{Discussion and conclusions}

\subsection{Remarks on the comparison process}

7.1.1. Photoionization. There were several issues regarding the implementation of photoionization. Different units and conventions are used in Luque's [66] and Bourdon's [55] approximations. Luque uses the total gas pressure, while
Bourdon uses the partial oxygen pressure. The proportionality factor $\xi$ is incorporated into Luque's parameters while one needs to multiply Bourdon's parameters by this factor (which here we set to $\xi=0.075$ ). Furthermore, only $\alpha$ should be used for the photoionization source term and not $\bar{\alpha}=\alpha-\eta$. Note that appendix A provides a detailed description of the formulas and parameters used for photoionization.

7.1.2. Oscillations and instabilities. Due to the low background ionization density of $n_{i}=n_{e}=10^{9} \mathrm{~m}^{-3}$ and the lack of photoionization, case 2 turned out to be highly challenging. No model got completely satisfactory solutions using each group's 'standard' numerical parameters (i.e. the parameters determined from experience to give good results). Oscillations in the electron density and electric field were observed, as well as streamer branching and numerical instabilities in some cases. Furthermore, we remark that a background electron density of $n_{e}=10^{9} \mathrm{~m}^{-3}=1 \mathrm{~mm}^{-3}$ is too low to get realistic results with an axisymmetric fluid model, as the continuum approximation (many electrons per fluid cell) is invalid and stochastic fluctuations are important.

7.1.3. Challenges in obtaining numerical convergence. As all groups solved the same equations with the same transport data, differences were either due to numerical discretization errors or due to bugs/flaws in (some of) the models. To distinguish between the two, we encouraged groups to perform simulations at higher and possibly also lower grid resolutions. However, clearly showing numerical convergence turned out to be surprisingly difficult, and in the end only the CWI, FR and DE groups participated in this effort. There were a couple of reasons for this:

- For models with AMR, there is no straightforward way to perform a convergence study, as errors in regions that are not refined (according to the refinement criterion) can eventually dominate.

- With a uniform grid, numerical convergence can more easily be studied, but for the test cases presented here (in a $(1.25 \mathrm{~cm})^{2}$ domain), having a high-resolution uniform grid is computationally challenging. The CWI and FR group therefore opted to refine only up to a certain radius.

- For the CN and DE models, simulations with standard grid resolution already took quite some time (e.g. a day), so that testing for numerical convergence was time consuming.

- Due to the strongly nonlinear growth of the streamer discharges and the steep gradients in electron and ion density, a grid spacing of about a micrometer was required before we got close to numerical convergence for case 1 . For case 2, which was computationally more difficult, even finer grids are required to reach the same level of convergence.

Because it was difficult to establish numerical convergence, it was also difficult to tell whether the differences were due to discretization errors or other causes. 
7.1.4. Discrepancies between $D E$ and $C N$. It should be noted that COMSOL is a multiphysics modeling environment, not streamer simulation software, and that it delivers the same results regardless of the user. COMSOL provides the possibility of (i) compiling the mathematical model to describe the physics, (ii) selecting the method for the spatial discretization of the mathematical model, (iii) selecting the time-discretization method, if applicable, and (iv) choosing a method for solutions of the nonlinear system of equations. In the present study, only step (i) was the same for all contributors. As for any other simulation software, whether self-written or commercial, steps (ii) to (iv) might affect the numerical solution as long as the numerical results do not approach the convergent solution.

\section{Conclusions}

In this study we compare six streamer simulation codes, using three test cases for axisymmetric positive streamers. The codes are of the finite volume or the finite element type, and they use both explicit and implicit time stepping. Simulations are performed in air at $300 \mathrm{~K}$ and $1 \mathrm{bar}$, and analytic expressions for the used transport coefficients are provided. The computational domain and initial conditions are kept simple, so other codes can be compared relatively easily to the results presented here. Supplementary data is also provided for this purpose.

The first test includes a homogeneous background density of $n_{i}=n_{e}=10^{13} \mathrm{~m}^{-3}$, and no photoionization. The results show quite good agreement when a sufficiently small grid spacing $\Delta x$ and time step $\Delta t$ are used. For codes with explicit time stepping, $\Delta t$ automatically decreases with $\Delta x$ due to stability constraints, whereas for implicit codes it had to be ensured that a sufficiently small $\Delta t$ was used. The second test case uses a lower background ionization level of $n_{e}=n_{i}=10^{9} \mathrm{~m}^{-3}$, which makes it computationally more challenging. With each group's standard numerical parameters (determined from experience to give good results), the models did not achieve completely satisfactory solutions. Oscillations in the electron density and electric field occurred, as well as branching and numerical instabilities in some cases. The oscillations disappeared for sufficiently small $\Delta x$ and $\Delta t$, and two models were able to reach reasonably good numerical convergence. The third test case is identical to the second one, except that photoionization is included based on Helmholtz approximations to Zhelenznyak's model. We compared different Helmholtz approximations, but found that for each group's standard numerical parameters, the numerical differences between models are more significant, and the results obtained with different photoionization parameters are very similar.

In summary, we find reasonably good agreement between models when sufficiently fine grids and corresponding small time steps are used, but not all participants were able to provide results close to numerical convergence. For the finite element codes included here, charge is only conserved when sufficiently small time steps are used. The computing times for the different models vary from minutes (for an explicit code with AMR) to days.
Table A1. Parameters by Luque et al [66].

\begin{tabular}{ccc}
\hline$j$ & $A_{j}^{L}\left(\mathrm{~cm}^{-2}\right.$ Torr $\left.^{-2}\right)$ & $\lambda_{j}^{L}\left(\mathrm{~cm}^{-1}\right.$ Torr $\left.^{-1}\right)$ \\
\hline 1 & $3.55 \times 10^{-6}$ & 0.010 \\
2 & $6.0 \times 10^{-5}$ & 0.059 \\
\hline
\end{tabular}

\section{Acknowledgments}

The authors thank Sergey Pancheshnyi for starting this comparison study, which originally aimed to directly compare experiment and simulation. BB acknowledges funding through the Dutch STW-project 15052, and JT through postdoctoral fellowship 12Q6117N of the Belgian-Flemish FWO. AL acknowledges support from European Research Council (ERC) under the European Union H2020 programme/ERC grant agreement 681257. The authors thank DANS (Data Archiving and Networked Services) for archiving the supplementary data.

\section{Appendix A. Photoionization}

The photoionization source term is given by the following integral:

$$
S_{\mathrm{ph}}(\mathbf{r})=\int \mathrm{d}^{3} r^{\prime} \frac{I\left(\mathbf{r}^{\prime}\right) f\left(\left|\mathbf{r}-\mathbf{r}^{\prime}\right|\right)}{4 \pi\left|\mathbf{r}-\mathbf{r}^{\prime}\right|^{2}}
$$

\section{A.1. Parametrization by Luque et al}

According to Luque et al [66], $I(\mathbf{r})$ is given by

$$
I(\mathbf{r})=\frac{p_{q}}{p+p_{q}} \xi^{L} S_{i}(\mathbf{r}),
$$

where $p_{q}$ is the quenching pressure. The factor $p_{q} /\left(p+p_{q}\right)$ accounts for the quenching of excited nitrogen molecules due to collisions, and we use a quenching pressure of $p_{q}=40$ mbar here. $S_{i}(\mathbf{r})=\alpha \mu_{e}|\mathbf{E}| n_{e}$ is the ionization source term. Note that $\alpha$ is the ionization coefficient without attachment $\eta$, not the effective ionization coefficient denoted in the main text as $\bar{\alpha} . \xi^{L}$ is the proportionality factor where we use the upper index $L$ to denote the $\xi$ of Luque's paper [66]. The absorption function $f$ is approximated as:

$$
f\left(\left|\mathbf{r}-\mathbf{r}^{\prime}\right|\right)=\frac{p^{2}\left|\mathbf{r}-\mathbf{r}^{\prime}\right|}{\xi^{L}} \sum_{j=1}^{N} A_{j}^{L} \mathrm{e}^{-\lambda_{j}^{L} p\left|\mathbf{r}-\mathbf{r}^{\prime}\right|} .
$$

Note that equation (A.3) is the corrected form of equation (2) in [66], and $p$ is the total pressure of air or gas mixture. We used the notation $A_{j}^{L}$ and $\lambda_{j}^{L}$ to indicate Luque's coefficients from [66]. By substituting equation (A.2) and equation (A.3) in equation (A.1), we get the following equation for the photoionization source term

$$
S_{\mathrm{ph}}(\mathbf{r})=\frac{p_{q}}{p+p_{q}} \sum_{j=1}^{N} p^{2} A_{j}^{L} \int d^{3} r^{\prime} \frac{S_{i}\left(\mathbf{r}^{\prime}\right) \mathrm{e}^{-\lambda_{j}^{L} p\left|\mathbf{r}-\mathbf{r}^{\prime}\right|}}{4 \pi\left|\mathbf{r}-\mathbf{r}^{\prime}\right|},
$$


Table A2. The parameters of two-term exponential fitting by Bourdon et al [55].

\begin{tabular}{ccc}
\hline$j$ & $A_{j}^{B}\left(\mathrm{~cm}^{-2}\right.$ Torr $\left.^{-2}\right)$ & $\lambda_{j}^{B}\left(\mathrm{~cm}^{-1}\right.$ Torr $\left.^{-1}\right)$ \\
\hline 1 & 0.0021 & 0.0974 \\
2 & 0.1775 & 0.5877 \\
\hline
\end{tabular}

Table A3. The parameters of three-term exponential fitting by Bourdon et al [55].

\begin{tabular}{ccc}
\hline$j$ & $A_{j}^{B}\left(\mathrm{~cm}^{-2}\right.$ Torr $\left.^{-2}\right)$ & $\lambda_{j}^{B}\left(\mathrm{~cm}^{-1}\right.$ Torr $\left.^{-1}\right)$ \\
\hline 1 & $1.986 \times 10^{-4}$ & 0.0553 \\
2 & 0.0051 & 0.1460 \\
3 & 0.4886 & 0.8900 \\
\hline
\end{tabular}

which can be solved with the following set of Helmholtz differential equations

$$
\begin{gathered}
\left(\nabla^{2}-\left(p \lambda_{j}^{L}\right)^{2}\right) S_{\mathrm{ph}, j}=-S_{i}, \\
S_{\mathrm{ph}}=\frac{p_{q}}{p+p_{q}} \sum_{j=1}^{N} p^{2} A_{j}^{L} S_{\mathrm{ph}, j}
\end{gathered}
$$

The coefficients $A_{j}^{L}$ and $\lambda_{j}^{L}$ are given in table $\mathrm{A} 1$, where $\xi^{L}$ is incorporated inside coefficients $A_{j}^{L}$.

\section{A.2. Parametrization by Bourdon et al}

Bourdon et al [55] used a different notation in their original paper. In equation (1) of the original paper, the function $g$ is used instead of $f$ in equation (A.1). Furthermore $I(\mathbf{r})$ is defined as

$$
I(\mathbf{r})=\frac{p_{q}}{p+p_{q}} \xi^{B} \frac{\nu_{u}}{\nu_{i}} S_{i}(\mathbf{r}),
$$

where $S_{i}(\mathbf{r})$ is the ionization source term and $\nu_{u}$ is the electron impact excitation frequency for level $u$. We assume that $\nu_{i}$ is the impact ionization rate. Note that here we use $\xi^{B}$ instead of $\xi$ in the original paper to denote Bourdon's values. In [55] the function $f$ is approximated as

$$
f\left(\left|\mathbf{r}-\mathbf{r}^{\prime}\right|\right)=p_{O_{2}}^{2}\left|\mathbf{r}-\mathbf{r}^{\prime}\right| \sum_{j=1}^{N} A_{j}^{B} \mathrm{e}^{-\lambda_{j}^{B} p_{O_{2}}\left|\mathbf{r}-\mathbf{r}^{\prime}\right|},
$$

where $p_{\mathrm{O}_{2}}$ is the partial pressure of oxygen molecules, and $A_{j}^{B}$ and $\lambda_{j}^{B}$ are Bourdon's coefficients (in the original paper they are $A_{j}$ and $\lambda_{j}$ ). By substituting equations (A.6) and (A.7) in (A.1) the photoionization source term reads

$$
\begin{aligned}
S_{\mathrm{ph}}(\mathbf{r})= & \frac{p_{q}}{p+p_{q}} \xi^{B} \frac{\nu_{u}}{\nu_{i}} \sum_{j=1}^{N} p_{O_{2}}^{2} A_{j}^{B} \\
& \times \int \mathrm{d}^{3} r^{\prime} \frac{S_{i}\left(\mathbf{r}^{\prime}\right) \mathrm{e}^{-\lambda_{j}^{B} p_{O_{2}}\left|\mathbf{r}-\mathbf{r}^{\prime}\right|}}{4 \pi\left|\mathbf{r}-\mathbf{r}^{\prime}\right|}
\end{aligned}
$$

which can be solved with the following set of Helmholtz
Table A4. A comparison of the two-term fittings by Bourdon et al [55] and by Luque et al [66] for dry air at 1 bar and $300 \mathrm{~K}$, for $\xi_{B} \frac{\nu_{u}}{\nu_{i}}=0.06$.

\begin{tabular}{ccccc}
\hline$j$ & $\lambda_{j}^{B} p_{O_{2}}$ & $\lambda_{j}^{L} p$ & $\xi^{B} \frac{\nu_{u}}{\nu_{i}} A_{j}^{B} p_{O_{2}}^{2}$ & $A_{j}^{L} p^{2}$ \\
\hline 1 & $14.610 \mathrm{~cm}^{-1}$ & $7.50 \mathrm{~cm}^{-1}$ & $2.835 \mathrm{~cm}^{-2}$ & $1.99 \mathrm{~cm}^{-2}$ \\
2 & $88.155 \mathrm{~cm}^{-1}$ & $44.25 \mathrm{~cm}^{-1}$ & $239.625 \mathrm{~cm}^{-2}$ & $33.75 \mathrm{~cm}^{-2}$ \\
\hline
\end{tabular}

differential equations

$$
\begin{aligned}
& \left(\nabla^{2}-\left(p_{O_{2}} \lambda_{j}^{B}\right)^{2}\right) S_{\mathrm{ph}, j}=-\left(A_{j}^{B} p_{O_{2}}^{2} \frac{p_{q}}{p+p_{q}} \xi^{B} \frac{\nu_{u}}{\nu_{i}}\right) S_{i}, \\
& S_{\mathrm{ph}}(\mathbf{r})=\sum_{j} S_{\mathrm{ph}}^{j}(\mathbf{r}) .
\end{aligned}
$$

The parameters for two-term and three-term exponential fitting are given in tables A2 and A3, respectively.

In the original paper by Zheleznyak et al [60], $\xi_{B} \frac{\nu_{u}}{\nu_{i}}$ is written as $\frac{\omega}{\alpha} \xi$, where $\omega$ is the number of ionizing photons created by an electron per $1 \mathrm{~cm}$ in the absence of a quenching process, $\alpha$ is the first Townsend ionization coefficient and $\xi$ is the average photoionization efficiency [60]. $\frac{\omega}{\alpha} \xi$ is given in table 1 in the original paper.

\section{A.3. Comparison of Luque's and Bourdon's fittings}

In summary, the parameters of the two-term fittings by Luque and Bourdon are related as follows:

$$
\lambda_{j}^{B} p_{O_{2}} \equiv \lambda_{j}^{L} p
$$

and

$$
\xi^{B} \frac{\nu_{u}}{\nu_{i}} A_{j}^{B} p_{O_{2}}^{2} \equiv A_{j}^{L} p^{2} .
$$

In our comparison instructions, we used $\xi_{B} \frac{\nu_{u}}{\nu_{i}}=0.075$, $p_{\mathrm{O}_{2}}=150$ Torr and $p=750$ Torr $=1$ bar. The physically equivalent parameters of the respective two-term fittings for dry air at $300 \mathrm{~K}$ are given in table $\mathrm{A} 4$, for $\xi_{B} \frac{\nu_{u}}{\nu_{i}}=0.06$.

\section{Appendix B. Description of supplementary data}

In this comparison study, each group performed a series of simulations and provided output files with the following information in time steps of $1 \mathrm{~ns}$ : maximum of electric field $E_{\max }$, the location of the maximal electric field $z_{\max }$, the total number of electrons $N_{e}$, the total charge in the system $Q$, the radius of the streamer head defined as the radius where the radial component of the electric field is maximal. Besides this, files including the axial $(r=0)$ data for the electric field strength and electron density at every 2 ns are provided. The input files together with the output data for each group are provided at: https://doi.org/10.17026/dans-x7r-266f. Moreover, the tabulated data for the transport and reaction coefficients used by CWI and ES are also included. 


\section{ORCID iDs}

B Bagheri (1) https://orcid.org/0000-0003-0887-5074

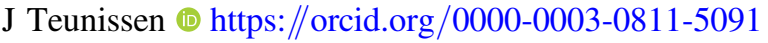
U Ebert $\odot$ https://orcid.org/0000-0003-3891-6869 M M Becker ํㅏ https://orcid.org/0000-0001-9324-3236 S Chen $\odot$ https://orcid.org/0000-0001-7013-1442 O Eichwald $\mathbb{1}$ i https://orcid.org/0000-0001-9941-4809 D Loffhagen 누 https://orcid.org/0000-0002-3798-0773 A Luque (ㄱ) https://orcid.org/0000-0002-7922-8627 M Yousfi (ㄴ) https://orcid.org/0000-0002-4979-2243

\section{References}

[1] Vitello P A, Penetrante B M and Bardsley J N 1994 Phys. Rev. E 49 5574-98

[2] Yi W J and Williams P F 2002 J. Phys. D: Appl. Phys. 35 205-18

[3] Ebert U, Nijdam S, Li C, Luque A, Briels T and van Veldhuizen E 2010 J. Geophys. Res. 115 A00E43

[4] Černák M, Kováčik D, St'ahel P, Zahoranová A, Kubincová J, Tóth A and Černáková L 2011 Plasma Phys. Control. Fusion 53124031

[5] Starikovskaia S M 2014 J. Phys. D: Appl. Phys. 47353001

[6] Starikovskii A Y, Nikipelov A A, Nudnova M M and Roupassov D V 2009 Plasma Sources Sci. Technol. 18 034015

[7] Joshi R P and Thagard S M 2013 Plasma Chem. Plasma Process. 33 17-49

[8] Jiang B, Zheng J, Qiu S, Wu M, Zhang Q, Yan Z and Xue Q 2014 Chem. Eng. J. 236 348-68

[9] Nozaki T and Okazaki K 2013 Catalysis Today 211 29-38

[10] Akiyama H 2000 IEEE Trans. Dielect. Electr. Insul. 7 646-53

[11] Kanazawa S, Kawano H, Watanabe S, Furuki T, Akamine S, Ichiki R, Ohkubo T, Kocik M and Mizeraczyk J 2011 Plasma Sources Sci. Technol. 20034010

[12] Nastuta A V, Topala I, Grigoras C, Pohoata V and Popa G 2011 J. Phys. D: Appl. Phys. 44105204

[13] Sands B L, Ganguly B N and Tachibana K 2008 Appl. Phys. Lett. 92151503

[14] Bekeschus S, Schmidt A, Weltmann K D and von Woedtke T 2016 Clinical Plasma Medicine 4 19-28

[15] Dhali S and Williams P 1985 Phys. Rev. A 31 1219-21

[16] Kulikovsky A A 1997 J. Phys. D: Appl. Phys. 30 441-50

[17] Babaeva N Y and Naidis G V 1996 J. Phys. D: Appl. Phys. 29 2423-31

[18] Pancheshnyi S V and Starikovskii A Y 2003 J. Phys. D: Appl. Phys. 36 2683-91

[19] Hagelaar G J M and Pitchford L C 2005 Plasma Sources Sci. Technol. 14 722-33

[20] Montijn C, Hundsdorfer W and Ebert U 2006 J. Comput. Phys. 219 801-35

[21] Luque A, Ebert U and Hundsdorfer W 2008 Phys. Rev. Lett. 101075005

[22] Eichwald O, Bensaad H, Ducasse O and Yousfi M 2012 J. Phys. D: Appl. Phys. 45385203

[23] Bessières D, Paillol J, Bourdon A, Ségur P and Marode E 2007 J. Phys. D: Appl. Phys. 40 6559-70

[24] Pancheshnyi S, Ségur P, Capeillère J and Bourdon A 2008 J. Comput. Phys. 227 6574-90

[25] Teunissen J and Ebert U 2017 J. Phys. D: Appl. Phys. 50 474001

[26] Plewa J M, Eichwald O, Ducasse O, Dessante P, Jacobs C, Renon N and Yousfi M 2018 J. Phys. D: Appl. Phys. 51 095206
[27] Zhuang C, Zeng R, Zhang B and He J 2014 IEEE Trans. Magn. 50 325-8

[28] Kulikovsky A 1995 J. Comput. Phys. 119 149-55

[29] Liu N and Pasko V P 2004 J. Geophys. Res. 109 A04301

[30] Bourdon A, Bonaventura Z and Celestin S 2010 Plasma Sources Sci. Technol. 19034012

[31] Morrow R and Lowke J J 1997 J. Phys. D: Appl. Phys. 30 614-27

[32] Georghiou G E, Morrow R and Metaxas A C 2000 J. Phys. D: Appl. Phys. 33 2453-66

[33] Kolobov V and Arslanbekov R 2012 J. Comput. Phys. 231 839-69

[34] Duarte M, Bonaventura Z, Massot M, Bourdon A, Descombes S and Dumont T 2012 J. Comput. Phys. 231 1002-19

[35] Celestin S, Bonaventura Z, Zeghondy B, Bourdon A and Ségur P 2009 J. Phys. D: Appl. Phys. 42065203

[36] Georghiou G, Morrow R and Metaxas A 1999 J. Comput. Phys. 148 605-20

[37] Ducasse O, Papageorghiou L, Eichwald O, Spyrou N and Yousfi M 2007 IEEE Trans. Plasma Sci. 35 1287-300

[38] O’Sullivan F, Hwang J, Zahn M, Hjortstam O, Pettersson L, Liu R and Biller P 2008 Conf. Record of the 2008 IEEE International Symposium on Electrical Insulation (Vancouver, BC, 9-12 June 2008) pp 210-14

[39] Papageorgiou L, Metaxas A C and Georghiou G E 2011 J. Phys. D: Appl. Phys. 44045203

[40] Zakari M, Caquineau H, Hotmar P and Ségur P 2015 J. Comput. Phys. 281 473-92

[41] Xiong Z and Kushner M J 2010 J. Phys. D: Appl. Phys. 43 505204

[42] Xiong Z and Kushner M J 2012 Plasma Sources Sci. Technol. 21034001

[43] Chanrion O and Neubert T 2008 J. Comput. Phys. 227 7222-45

[44] Rose D V, Welch D R, Clark R E, Thoma C, Zimmerman W R, Bruner N, Rambo P K and Atherton B W 2011 Phys. Plasmas 18093501

[45] Teunissen J and Ebert U 2016 Plasma Sources Sci. Technol. 25 044005

[46] Kolobov V and Arslanbekov R 2016 J. Phys. Conf. Series 719 012020

[47] Jiang M, Li Y, Wang H and Liu C 2017 Phys. Plasmas 24 102112

[48] Li C, Ebert U and Hundsdorfer W 2012 J. Comput. Phys. 231 1020-50

[49] Babaeva N Y, Tereshonok D V and Naidis G V 2016 Plasma Sources Sci. Technol. 25044008

[50] Roache P J 1998 Verification and Validation in Computational Science and Engineering Vol 895 (Socorro, NM: Hermosa) p 895

[51] Turner M M, Derzsi A, Donkó Z, Eremin D, Kelly S J, Lafleur T and Mussenbrock T 2013 Phys. Plasmas 20 013507

[52] Kim H C, Iza F, Yang S S, Radmilović-Radjenović M and Lee J K 2005 J. Phys. D: Appl. Phys. 38 R283-301

[53] Li C, Teunissen J, Nool M, Hundsdorfer W and Ebert U 2012 Plasma Sources Sci. Technol. 21055019

[54] Markosyan A H, Teunissen J, Dujko S and Ebert U 2015 Plasma Sources Sci. Technol. 24065002

[55] Bourdon A, Pasko V P, Liu N Y, Célestin S, Ségur P and Marode E 2007 Plasma Sources Sci. Technol. 16 656-78

[56] Pancheshnyi S, Nudnova M and Starikovskii A 2005 Phys. Rev. E 71016407

[57] Celestin S, Bonaventura Z, Guaitella O, Rousseau A and Bourdon A 2009 Eur. Phys. J. Appl. Phys. 4722810

[58] Dubinova A, Trienekens D, Ebert U, Nijdam S and Christen T 2016 Plasma Sources Sci. Technol. 25055021 
[59] Briels T M P, Kos J, Winands G J J, van Veldhuizen E M and Ebert U 2008 J. Phys. D: Appl. Phys. 41234004

[60] Zhelezniak M, Mnatsakanian A and Sizykh S 1982 High Temp. 20 357-62

[61] Ebert U, Saarloos W V and Caroli C 1997 Phys. Rev. E 55 1530-49

[62] Derks G, Ebert U and Meulenbroek B 2008 J. Nonlinear Sci. 18 551-90

[63] Roy C J 2005 J. Comput. Phys. 205 131-56

[64] Dutton J 1975 J. Phys. Chem. Ref. Data 4 577-856

[65] Hartmann G 1984 IEEE Trans. Ind. Appl. IA-20 1647-51

[66] Luque A, Ebert U, Montijn C and Hundsdorfer W 2007 Appl. Phys. Lett. 90081501

[67] Teunissen J and Ebert U 2018 Comput. Phys. Commun. 233 $156-66$
[68] Luque A and Ebert U 2012 J. Comput. Phys. 231 904-18

[69] van Dijk J, Peerenboom K, Jimenez M, Mihailova D and van der Mullen J 2009 J. Phys. D: Appl. Phys. 42 194012

[70] Ventzek P, Hoekstra R and Kushner M 1994 J. Vac. Sci. Technol. B 12 461-77

[71] Becker M M, Kählert H, Sun A, Bonitz M and Loffhagen D 2017 Plasma Sources Sci. Technol. 26044001

[72] Becker M M, Hoder T, Brandenburg R and Loffhagen D 2013 J. Phys. D: Appl. Phys. 46355203

[73] Sigeneger F, Becker M M, Foest R and Loffhagen D 2016 J. Phys. D: Appl. Phys. 49345202

[74] Demmel J W, Eisenstat S C, Gilbert J R, Li X S and Liu J W H 1999 SIAM J. Matrix Anal. Appl. 20 720-55 